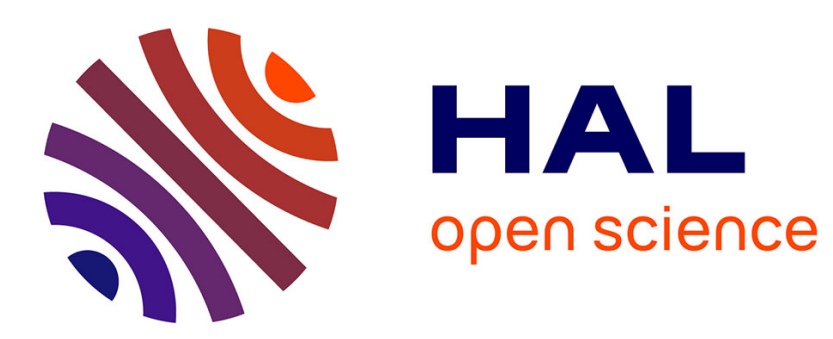

\title{
Two- and three-dimensional numerical simulations of the transition to oscillatory convection in low-Prandtl-number fluids
}

Daniel Henry, Marc Buffat

\section{- To cite this version:}

Daniel Henry, Marc Buffat. Two- and three-dimensional numerical simulations of the transition to oscillatory convection in low-Prandtl-number fluids. Journal of Fluid Mechanics, 1998, 374, pp.145171. 10.1017/S0022112098002523 . hal-00123707

\section{HAL Id: hal-00123707 https://hal.science/hal-00123707}

Submitted on 18 Jan 2007

HAL is a multi-disciplinary open access archive for the deposit and dissemination of scientific research documents, whether they are published or not. The documents may come from teaching and research institutions in France or abroad, or from public or private research centers.
L'archive ouverte pluridisciplinaire HAL, est destinée au dépôt et à la diffusion de documents scientifiques de niveau recherche, publiés ou non, émanant des établissements d'enseignement et de recherche français ou étrangers, des laboratoires publics ou privés. 


\title{
Two- and three-dimensional numerical simulations of the transition to oscillatory convection in low-Prandtl-number fluids
}

\author{
By DANIEL HENRY AND MARC BUFFAT \\ Laboratoire de Mécanique des Fluides et d'Acoustique-UMR CNRS 5509, Ecole Centrale de \\ Lyon/Université Claude Bernard-Lyon 1, ECL, BP 163, 69131 Ecully Cedex, France
}

(Received 24 May 1991 and in revised form 1 June 1998)

The convective flows which arise in shallow cavities filled with low-Prandtl-number fluids when subjected to a horizontal temperature gradient are studied numerically with a finite element method. Attention is focused on a rigid cavity with dimensions $4 \times$ $2 \times 1$, for which experimental data are available. The three-dimensional results indicate that, after a relative concentration of the initial Hadley circulation, a transition to time-dependent flows occurs in the form of a roll oscillation with a purely dynamical origin. This transition corresponds to a Hopf bifurcation with a breaking of symmetry that gives some specific properties to the time evolution of the flow: these properties are shown to be the result of the general behaviour of the dynamical systems. Calculations performed in the case of mercury compare well with the experiments with similar power spectra of the temperature, and this validates the analysis of the nature of the global flow performed in the limiting case $P r=0$. All these results are discussed with respect to the linear and nonlinear analyses and to other computational experiments. Numerical results obtained in the corresponding twodimensional situation give a different transition to the time-dependent flow: it is shown that in the three-dimensional cavity this type of two-dimensional transition is less probable than the observed transition with breaking of symmetry.

\section{Introduction}

During the growth of metal and semi-conductor crystals (like Ga-As) from a liquid melt in horizontal boat (e.g. by the Bridgman technique), undesirable striations corresponding to an irregular distribution of solute in the crystal may occur. It has been shown that such striations are caused by spontaneous temperature oscillations generating fluctuations in the rate of growth of the crystal (see the survey paper of Pimputkar \& Ostrach 1981).

Experiments without solidification carried out for pure molten Ga in cavities with a horizontal temperature gradient (Hurle, Jakeman \& Johnson 1974) exhibit such oscillations. Thus, in a cavity with differentially heated vertical endwalls (at temperatures $T_{1}$ and $T_{2}$ ), a buoyancy flow occurs as soon as $T_{1}$ is different from $T_{2}$. For small temperature difference $\Delta T=T_{2}-T_{1}$, this flow corresponds to a simple unicellular flow, where motion is up the hot wall, across the top, down the cold wall and returning across the bottom, and is known as a Hadley circulation. But when $\Delta T$ is increased beyond a critical value $\Delta T_{c}$ (corresponding to a critical Grashof number $G r_{c}$ ), fluids with a low Prandtl number $P r$ present the onset of oscillations experimentally registered on the temperature. 
An attempt to explain these oscillations has been done by linear stability analysis applied on the basic unicellular Hadley circulation. After the work of Hart (1972) showing the possible occurrence of a longitudinal oscillatory instability of this basic state, Gill (1974) suggested that this instability is the cause of the experimentally observed oscillations and gave a physical description of the nature of these oscillations. More recently, Hart (1983a), Laure (1987) and Laure \& Roux (1987) extended this work by considering different boundary conditions. Then Laure (1987), Laure \& Roux (1987), Kuo \& Korpela (1988) and Wang \& Korpela (1989) obtained new information by using a nonlinear analysis. These results, although very interesting, are perhaps not relevant for the problem under consideration: as shown numerically by Cormack, Leal \& Imberger (1974) and more recently by Hart (1983b), Dupont et al. (1987) and at the GAMM workshop (Roux 1990), the Hadley circulation is not the appropriate basic flow for the domain of values of $G r$ at which oscillations occur. An alternative approach, in which the perturbation is imposed on the basic steady flow (continuation method), has been used by Winters (1988) for the $4 \times 1$ box, and by Skeldon, Riley \& Cliffe (1996) who extended the work by varying the aspect ratio and the inclination of the box. However, this approach has up to now mainly applied to two-dimensional situations. Analysis of the secondary instabilities (those affecting the transverse rolls) has been performed by Wang \& Korpela (1992), but their results do not explain the characteristics of the experimentally observed oscillatory instabilities.

The numerical simulation of oscillatory regimes in low- $\mathrm{Pr}$ fluids has given valuable information. After the works of Crochet, Geyling \& Van Schaftingen (1983, 1987), Roux, Bontoux \& Henry (1985), Ben Hadid \& Roux (1987), showing the existence of oscillatory regimes in two-dimensional rectangular cavities, the two-dimensional simulations of the GAMM workshop (Roux 1990) in a $4 \times 1$ box agreed on the threshold value of $\mathrm{Gr}$ for the onset of oscillations and on the frequency of these oscillations (close to the values obtained by Winters 1988), and compared roughly with the results of Hurle et al. (1974), Hung \& Andereck (1988, 1990), Hart \& Pratte (1990) and Pratte \& Hart (1990). But it is not known whether the processes found for two-dimensional simulations are relevant to explain the oscillations obtained in real three-dimensional cavities.

Three-dimensional computations were necessary for a better understanding of these phenomena. Some have been carried out by Dupont et al. (1987) in the case of open cavities, by Roux et al. (1985) in the case of a cylinder, and by different contributors to the GAMM workshop (Roux 1990), but they were all limited to stationary results. The experimental results obtained by Hung \& Andereck (1990), Hart \& Pratte (1990) and Pratte \& Hart (1990) in the case of $4 \times 2 \times 1$ and $4 \times 1 \times 1$ rigid cavities with adiabatic lateral walls, motivated dealing with such situations. Another experimental investigation by McKell et al. (1990) concentrated rather on the chaotic behaviour, whereas a recent work by Braunsfurth \& Mullin (1996) investigated the variation of the onset of oscillation as a function of the Prandtl number. Afrid \& Zebib (1990) solved the three-dimensional problem, but they assumed a symmetry with respect to the longitudinal vertical plane even in the oscillatory case, which is in opposition with the experimental results (Pratte \& Hart 1990). More recently, Mundrane \& Zebib $(1993,1994)$ analysed combined buoyancy- and thermocapillary-driven convection, respectively in the steady regime for three-dimensional configurations and in the oscillatory regime for two-dimensional configurations.

We chose to consider the case of a rigid cavity with adiabatic lateral walls and $4 \times 2 \times 1$ dimensions which, up to now, has not really been resolved. The values of 


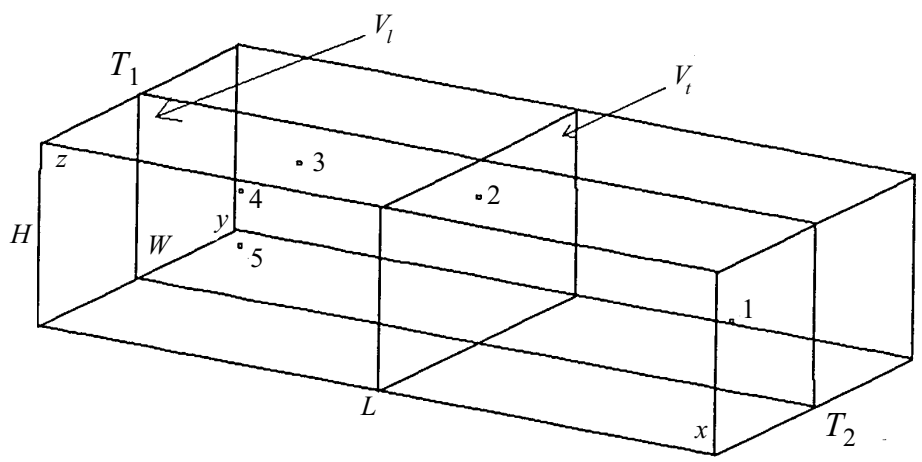

FIGURE 1. Differentially heated cavity: definition sketch for dimensions, coordinates, main planes and locations of points $P_{1}-P_{5}$ (labelled 1-5) where time variations are recorded: $P_{1}=(3.697,0.678,0.5)$, $P_{2}=(2,1,0.795), P_{3}=(0.942,1,0.795), P_{4}=(0.942,0.4098,0.795), P_{5}=(0.942,0.4098,0.5)$.

$G r$ are taken up to 45000 , whereas the values of $\operatorname{Pr}$ correspond mainly to mercury $(P r=0.026)$, together with the limiting case $P r=0$. The three-dimensional results are analysed and compared with the different experimental results available: they show good agreement based on different characteristics of the flow and of the oscillatory behaviour. A synthesis of the results obtained in the corresponding two-dimensional cavity $(4 \times 1)$ is also given in order to allow comparisons between the oscillatory regimes in two-dimensional and three-dimensional situations.

\section{Posing the problem and equations}

We consider a parallelepipedic rigid cavity having two differentially heated endwalls at temperatures $T_{1}$ and $T_{2}\left(T_{2}>T_{1}\right)$, and filled up with a low-Prandtl-number fluid (see figure 1). The dimensions of the cavity are the length $L$, the width $W$ and the height $H$ on the axes $o_{x}, o_{y}$ and $o_{z}$ respectively. We can then define two aspect ratios: $A_{x}=L / H$ and $A_{y}=W / H\left(A_{x} \times A_{y} \times 1\right.$ cavity $)$. The two-dimensional situation concerns the middle longitudinal vertical plane $\left(V_{l}\right.$ plane parallel to $\left.\left(o_{x}, o_{z}\right)\right)$ and is characterized by the length $L$ and the height $H$ or the aspect ratio $A_{x}\left(A_{x} \times 1\right.$ cavity).

We assume that the velocity is small enough to consider the flow as laminar. In addition, the fluid is supposed to be Newtonian and quasi-incompressible (Boussinesq approximation): the physical properties (kinematic viscosity $v$, diffusivity $\kappa$ and density $\bar{\rho}$ ) are assumed to be constant, except in the buoyancy term where $\bar{\rho}$ is taken as a linear function of the temperature $\bar{T}$ :

$$
\bar{\rho}=\rho_{1}\left(1-\alpha\left(\bar{T}-T_{1}\right)\right)
$$

where $\alpha$ is the thermal expansion coefficient (an overbar indicates a dimensional variable).

The motion of the fluid is then governed by the incompressible Navier-Stokes equations coupled with the energy equation. With reference length, time, velocity, pressure and temperature given respectively by $H, H^{2} / v, G r^{0.5} v / H, G r^{0.5} \rho v^{2} / H^{2}$ and $\bar{T}_{r e f}=\Delta \bar{T} / A_{x}$, we obtain the following set of dimensionless equations expressed in terms of the velocity vector $\boldsymbol{u}(u, v, w)$, the pressure $p$ and the temperature $T$ :

$$
\nabla \cdot \boldsymbol{u}=0
$$




$$
\begin{gathered}
\frac{\partial \boldsymbol{u}}{\partial t}+G r^{0.5}(\boldsymbol{u} \cdot \nabla) \boldsymbol{u}=-\nabla p+G r^{0.5} T \boldsymbol{e}_{z}+\nabla^{2} \boldsymbol{u}, \\
\frac{\partial T}{\partial t}+G r^{0.5} \boldsymbol{u} \cdot \nabla T=\frac{1}{P r} \nabla^{2} T .
\end{gathered}
$$

The non-dimensional parameters are the Grashof number $G r=\alpha g \Delta \bar{T} H^{3} / A_{x} v^{2}$ and the Prandtl number $\operatorname{Pr}=v / \kappa$. The dimensionless temperature is chosen as $T=$ $\left(\bar{T}-T_{1}\right) / \bar{T}_{r e f}$. The use of $G r^{0.5} v / H$ for the dimensionless velocity is recommended by Ostrach (1976) for large values of $\mathrm{Gr}$ (where inertia balances buoyancy). The associated boundary conditions are:

$u=0, v=0, w=0$ on all the boundaries (rigid walls);

$T=0$ at $x=0$ (cold vertical wall);

$T=A_{x}$ at $x=A_{x}$ (hot vertical wall);

$\partial T / \partial n=0$ (adiabatic condition) on the horizontal and vertical longitudinal walls.

In the limiting case $P r=0$ (highly conducting fluid with a finite $v$ and an infinite $\kappa)$, (2.4) becomes $\nabla^{2} T=0$. Taking into account the boundary conditions, the solution corresponds to the diffusive temperature profile $T=T_{0}(x)=x$. The system (2.2)-(2.4) is then reduced to

$$
\begin{gathered}
\nabla \cdot \boldsymbol{u}=0 \\
\frac{\partial \boldsymbol{u}}{\partial t}+G r^{0.5}(\boldsymbol{u} \cdot \nabla) \boldsymbol{u}=-\nabla p+G r^{0.5} x \boldsymbol{e}_{z}+\nabla^{2} \boldsymbol{u} .
\end{gathered}
$$

To summarize, the configurations under study correspond to a rigid cavity with adiabatic lateral walls and $4 \times 2 \times 1$ dimensions. Some two-dimensional simulations are performed in the corresponding two-dimensional geometry $(4 \times 1)$. The main simulations correspond to the limiting case $P r=0$, but also to $P r=0.026$ in order to study the effect of $P r$ and to compare with the experimental results.

\section{Numerical method and characteristics of the calculations}

The general system (2.2)-(2.4) is solved with a computer code developed by Buffat (1991a) for the simulation of two- and three-dimensional subsonic flows. The original time integration is based on a first-order semi-implicit scheme which allows for the linearization of the equations and the formal decoupling between the energy equation and the Navier-Stokes equations. However this scheme is too diffusive in time and we have replaced it by an implicit second-order scheme to solve precisely and efficiently (i.e. without too small time steps) non-stationary flows (Buffat 1991b).

The space discretization is based on a finite element method which can deal with complex geometries; this could be useful for the simulation of real crystal growth configurations. The finite element used is the $P^{1} /$ iso $P^{2}$ element which gives a continuous and piecewise linear interpolation for the pressure associated with a continuous and piecewise linear interpolation for the velocity components and the temperature on a grid twice as fine as the pressure grid.

The two-dimensional simulations have been performed on a regular mesh which is symmetrical with respect to the centre of the cavity and has $97 \times 41$ nodes. The three-dimensional results have been obtained with a slightly graded mesh which is symmetrical with respect to the main centre planes and has $57 \times 17 \times 17$ nodes. To test the grid dependence of the numerical results, we have done again some stationary calculations on a refined mesh with $73 \times 25 \times 25$ grid points. In the stationary case at $P r=0$ and $G r=30000$, the maximal difference on the nodal values of the velocity components is less than $5 \%$, showing that the mesh used in this study is a 
little too coarse in some parts of the cavity but retains the main characteristics of the flow. The calculations have been realized with the second-order scheme with a time step $\Delta t$ between $10^{-3}$ and $5 \times 10^{-4}$, which allows a precise representation of the oscillatory solutions with about a hundred of time steps per period. A general transient evolution requires a few dozen periods or a dimensionless time of about 1 . To allow an estimation of the times involved in a typical experimental situation, the dimensional reference time, $\mathrm{H}^{2} / v$, corresponds to values around $558 \mathrm{~s}$ and $872 \mathrm{~s}$ for respectively the experiments of Hung \& Andereck (1990) and Hart \& Pratte (1990).

The results in the three-dimensional case are generally presented by views in the principal middle planes (figure 1): the longitudinal vertical plane $\left(V_{l}\right.$ plane, plane of symmetry for low $G r)$, the longitudinal horizontal plane $\left(H_{l}\right.$ plane) and the transversal vertical plane $\left(V_{t}\right.$ plane). Some three-dimensional views of particles tracks are shown. Time variations recorded for the velocity components and for the temperature at different points $P_{1}-P_{5}$ inside the cavity (see figure 1) are also given.

\section{Two-dimensional results}

The problem of the two-dimensional rigid cavity with dimensions $4 \times 1$ has been treated in detail by many authors during the GAMM workshop (Roux 1990) (see also Pulicani et al. 1990; Bontoux et al. 1990). Our objective in this section is to make a synthesis of the results available with stress on some particular properties in order to compare with the three-dimensional results.

At small Grashof numbers, a parallel flow circulation with recirculation at the ends (Hadley circulation) is obtained. When increasing the Grashof number, as mentioned by Hart (1983b) and confirmed by Drummond \& Korpela (1987), this Hadley circulation is first modified by inertial effects near the ends of the cavity leading to the creation of small cat's eye vortices in these parts of the cavity. These two end vortices grow regularly and lead to the successive build-up of eddies from the ends to the centre, and thus as shown by Hart (1983b) to a smooth transition (imperfect bifurcation) to the steady multi-vortices pattern predicted by the linear stability analysis (Hart 1983a; Laure 1987). For the aspect ratio $A_{x}=4$, the two small cat's eye vortices, visible for $G r=5000$, quickly merge, giving a single centre vortex that becomes stronger and begins to tilt as $\mathrm{Gr}$ is increased. This centre vortex becomes concentrated, leading to a more and more well defined convective cell as it gets stronger vertical velocities (figure 2), and this enables the formation of small recirculation patterns near the ends (Roux 1990). Such small eddies are obtained by Drummond \& Korpela (1987) in larger cavities in the region between the cells and are interpreted as a secondary instability. It seems that, as the Grashof number increases, the concentration of the original cells leaves enough space between the rolls to enable the creation by shear of small vortices. All these stationary results are symmetric with respect to the centre of the cavity.

The further behaviour of the flow for increasing $G r$ has been obtained during the GAMM workshop (Roux 1990), and completed by Pulicani et al. (1990) and Bontoux et al. (1990). Above a critical value of the Grashof number, $G r_{c}$, given by Le Quéré (1990) as $G r_{c}=25350$ for $P r=0$, an oscillatory regime is obtained. By increasing the Grashof number, there is a succession of various regimes: monoperiodic, quasi-periodic, with even period doubling behaviour (Pulicani et al. 1990). In all these oscillatory cases, the flow corresponds to oscillations of the three steady rolls obtained at smaller Grashof number. A further increase in $G r$ leads, for a value close to $G r=35000$, to a reverse transition to stationary convection with 


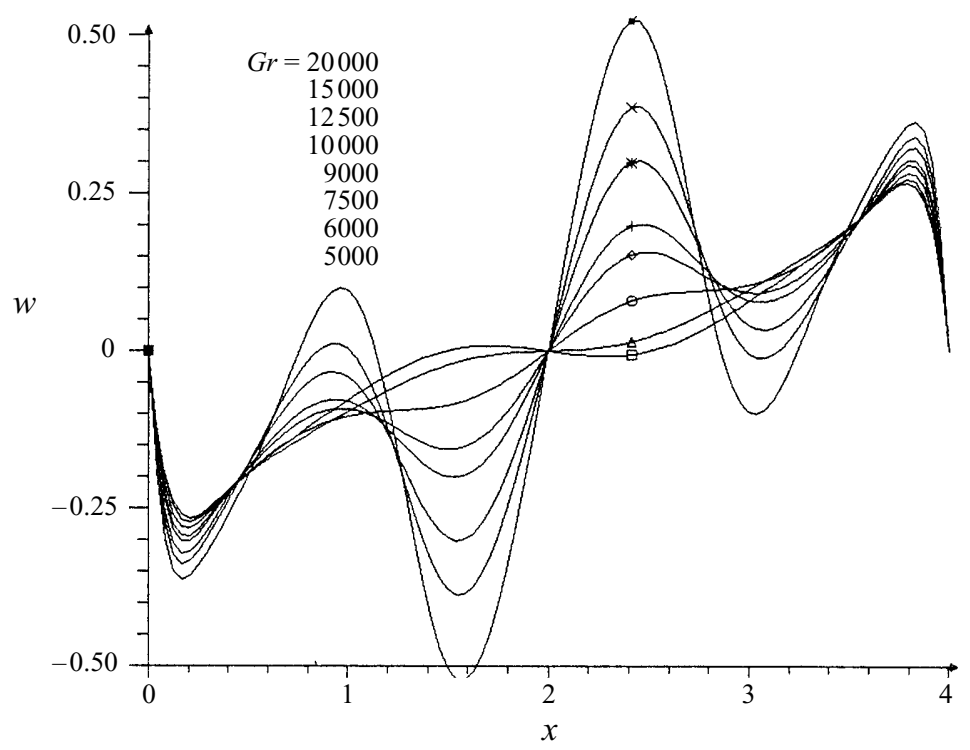

FIGURE 2. Vertical velocity $(w)$ profiles as a function of $x$ for $z=0.5$. Two-dimensional steady cases in the $4 \times 1$ cavity for $P r=0$ and different values of $G r$.

two almost independent rolls filling the whole height of the cavity ('flywheel' or inertial convection roll mentioned for long cavities by Drummond \& Korpela (1987) and Roux, Ben Hadid \& Laure (1989), and precisely studied for Rayleigh-Bénard convection by Clever \& Busse (1981) and Busse \& Clever (1981)). In fact, this two-roll structure belongs to another solution branch which arises through a saddle-node point at a threshold very close to $G r_{c}$ (Winters 1988; Bontoux et al. 1990). This indicates that this stationary two-cell regime coexists with the oscillatory three-cell regimes, and suggests that the oscillations correspond to a competition between the rolls due to geometric constraints: the length of the cavity is too short for three equally developed rolls to settle in such a cavity.

\section{Three-dimensional results}

The simulation of the three-dimensional flow in a parallelepipedic rigid box has been performed for dimensions corresponding to $A_{x}=4$ and $A_{y}=2(4 \times 2 \times 1$ box $)$. The general study will concern the limiting case $\operatorname{Pr}=0$, before analysing the effect of $P r$.

\subsection{Stationary convection $(\operatorname{Pr}=0)$}

An evolution from rest gives for $G r=10000$ a stationary one-cell convective state. Such a stationary state is still obtained for larger Grashof numbers, as a stable state for $G r=20000$, and as an unstable state for still larger $G r$ values $(30000$, $40000,45000)$. In all these cases, we observe the two symmetries mentioned by Laure (1987) for a rigid cavity: a symmetry with respect to the $V_{l}$ plane (called $S$ symmetry and corresponding to a reflection about the $V_{l}$ plane), and a symmetry with respect to the middle horizontal transversal axis $H_{t}(x=2, z=0.5)$ (called $S_{r}$ symmetry and corresponding to a reflection about the $H_{t}$ axis). The combination of these two symmetries gives a symmetry with respect to the centre point of the cavity (noted $S_{c}$ symmetry). 
(a)

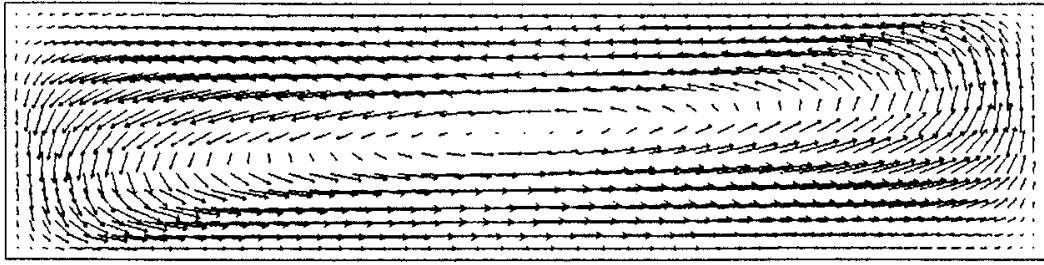

(b)

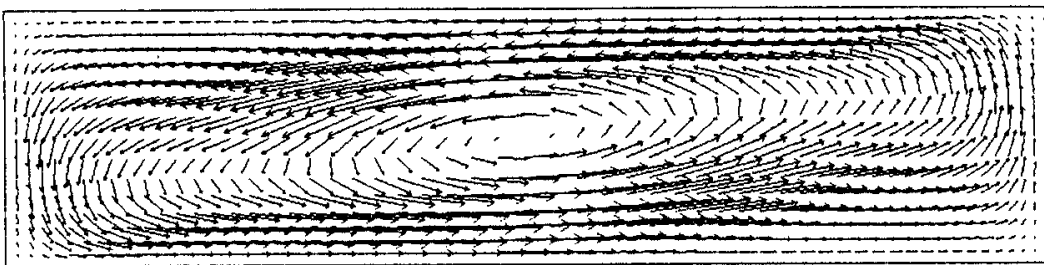

FiguRE 3. Steady flow solution in the $4 \times 2 \times 1$ cavity for $\operatorname{Pr}=0$. Velocity vector fields in the $V_{l}$ plane: (a) $G r=10000$; (b) $G r=20000$.

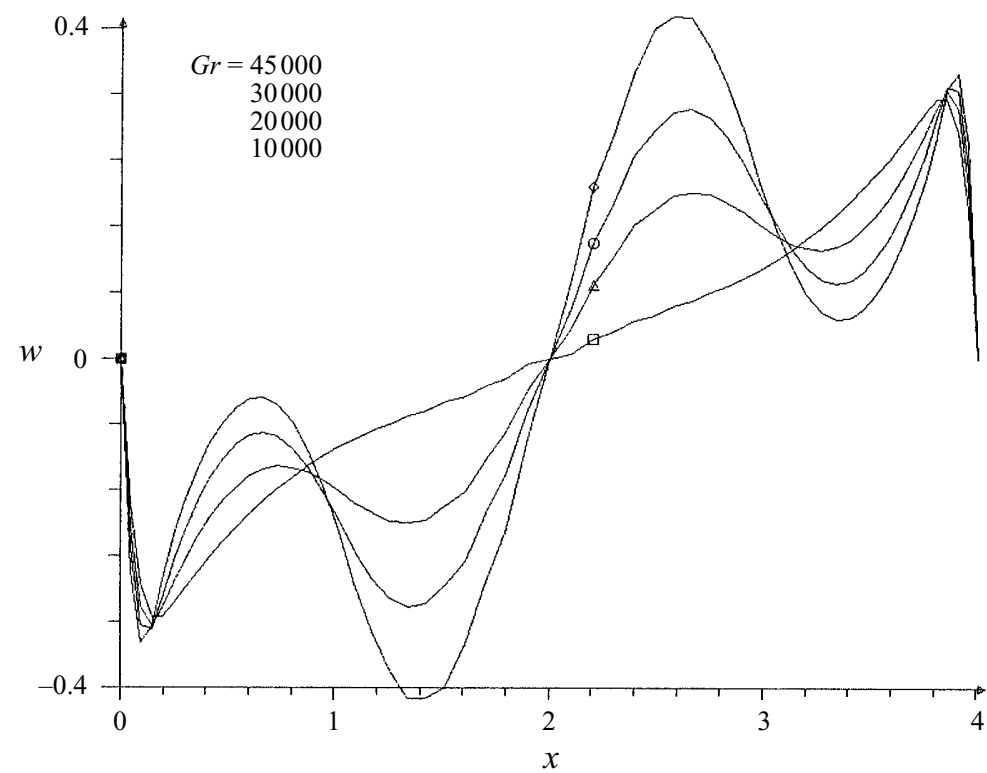

FIGURE 4. Steady flow solution in the $4 \times 2 \times 1$ cavity for $\operatorname{Pr}=0$. Vertical velocity $(w)$ profiles in the $V_{l}$ plane at $z=0.5$ for different values of $G r$.

As shown in figure $3(a)$, the flow at $G r=10000$ corresponds to a single roll, already tilted compared to the Hadley circulation. This roll seems to concentrate in the core of the cavity at higher Grashof numbers (figure $3 b$ ). This effect is confirmed by the vertical velocity profiles given in figure 4 which show a well defined central roll, clearly distinct from the peripheral long-scale circulation. These profiles compare well with the ones obtained in figure 2 for the two-dimensional simulations (see for instance the curves corresponding to $G r=6000,9000,10000$ and 12500). This suggests that an imperfect bifurcation, similar to the multi-vortices imperfect bifurcation obtained in the two-dimensional simulations, begins to develop in the three-dimensional cavity, but it occurs at a larger Grashof number, and even up to $G r=45000$ the small eddies near the ends are not obtained. The appearance of this steady bifurcation 

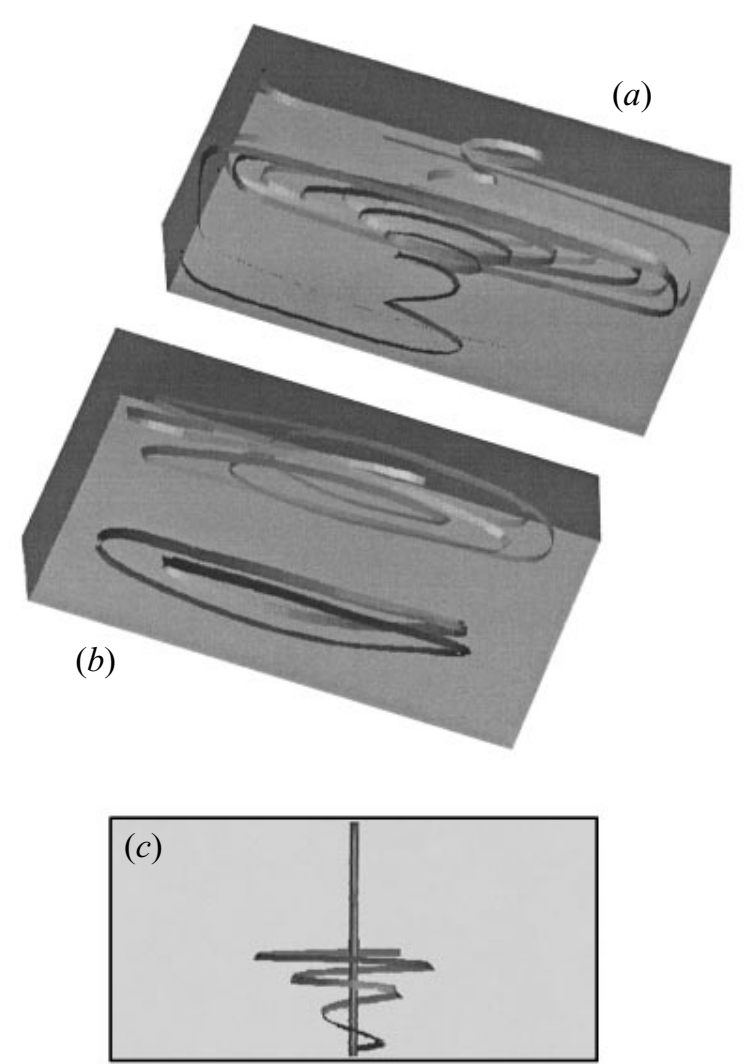

FIGURE 5. Steady flow solution in the $4 \times 2 \times 1$ cavity for $P r=0$ and $G r=20000$ : (a) particle tracks issued from the points $(0.1,0.1,0.5)$ and $(0.1,1.9,0.5) ;(b)$ particle tracks issued from the points $(3,0.5,0.4)$ and $(1,1.5,0.75) ;(c)$ central vorticity tube passing through the centre of the cavity and particle track issued from the point $(2,0.1,0.35)$.

before any oscillatory behaviour is in agreement with the stability analysis of the Hadley circulation (Hart 1972; Laure 1987; Kuo \& Korpela 1988) which predicts the first transition for $\mathrm{Pr}=0$ as a stationary mode with tranverse rolls. Moreover, according to the two more accurate studies (Laure 1987; Kuo \& Korpela 1988) this stationary transition will occur first up to $P r=0.033$, which includes the case of the experiments on mercury with $\operatorname{Pr}=0.026$.

Important three-dimensional effects are present in these steady situations. They transform the principal unicellular circulation in planes parallel to the $V_{l}$ plane to a spiralling motion in the half-cavities between the lateral walls and the $V_{l}$ plane (the $S$ symmetry prevents the flow going through the $V_{l}$ plane). According to the particle tracks given in figure $5(a-c)$, the particles spiral from the lateral wall towards the $V_{l}$ plane with an increasing radius, return quite quickly to the lateral wall by a large spiral along the walls and then flow inwardly quickly near the lateral walls. This spiralling motion occurs mainly in the region of the cavity composed of a conical domain near the $H_{t}$ axis and the domain along the $V_{l}$ plane and the wall boundaries. For particles taken outside this region in the intermediate torus (figure $5 b$ ), the spiralling motion is less pronounced. All this is strongly connected with the four vortices observed in the horizontal $H_{l}$ plane. These results look like those obtained by Mallinson \& de Vahl Davis (1977) in similar cavities heated from the side but with different aspect ratios, 


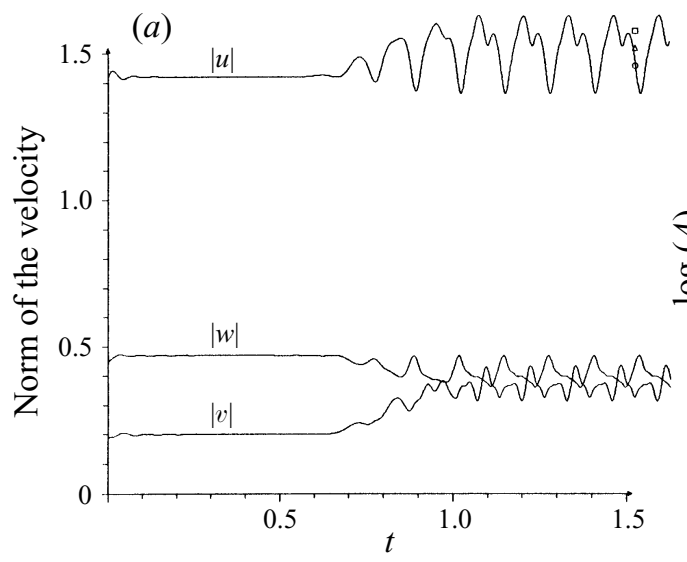

(b)
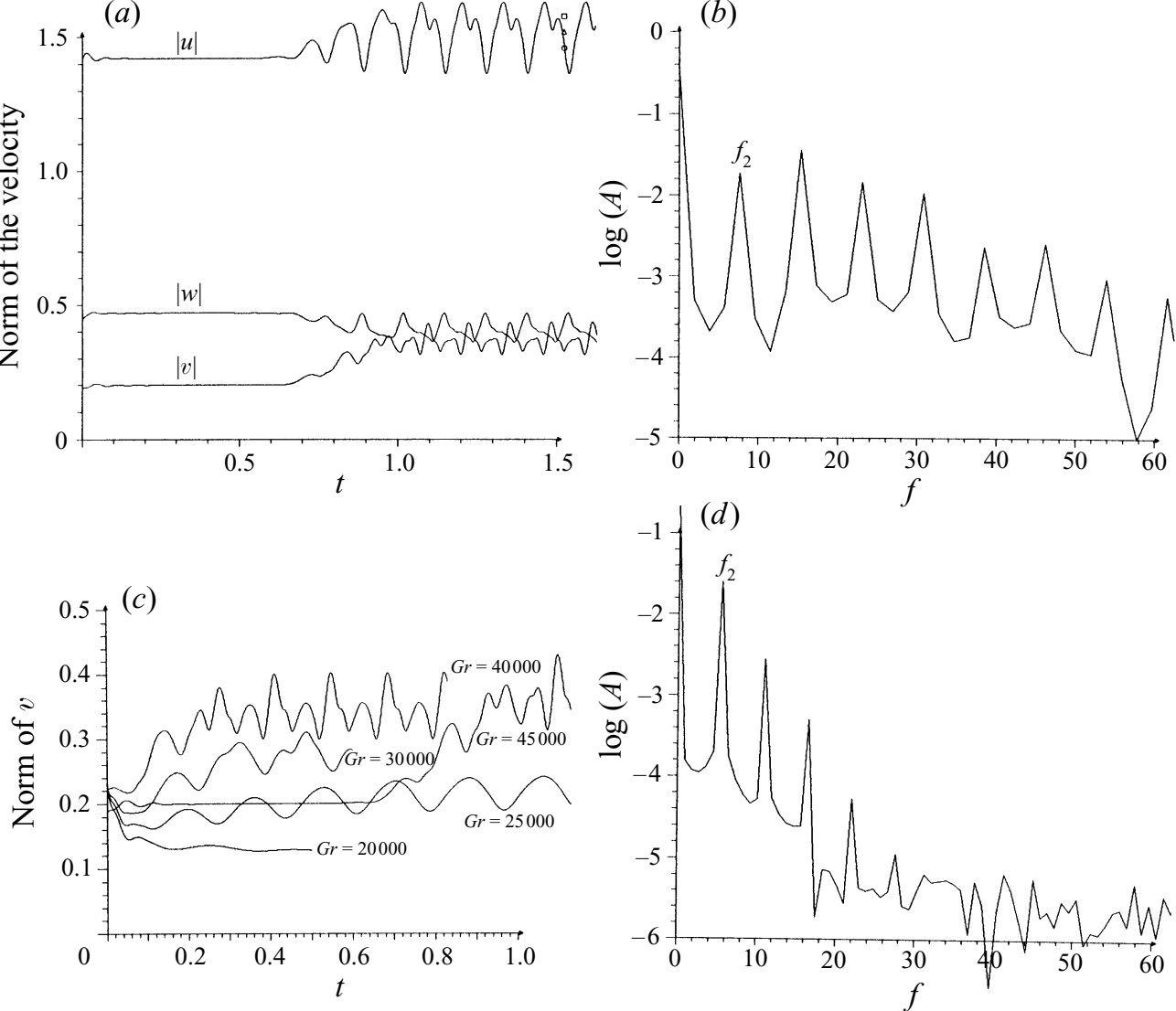

FiguRE 6. Transition from the steady to the oscillatory flow in the $4 \times 2 \times 1$ cavity for $\operatorname{Pr}=0$ : $(a)$ evolution with time of the $L^{2}$ norm of the velocity components for $G r=45000$ : destabilization of the unstable steady state; $(b)$ corresponding power spectrum of the $L^{2}$ norm of $v ;(c)$ evolution with time of the $L^{2}$ norm of $v$ for different $G r$, with a same initial perturbed state corresponding to the convective state for $G r=45000$ at $t=0.7$ (see $a) ;(d)$ corresponding power spectrum of the $L^{2}$ norm of $v$ for $G r=25000$.

where the spiralling motion was explained as the result of the interaction between the rotating flow and the wall.

\subsection{Oscillatory convection}

A transition to oscillatory convection is observed for large values of $G r$. For $G r=$ 45000 , growing perturbations have naturally destabilized the unstable steady flow (figure $6 a$ ) whereas for lower $G r(G r=40000,30000$ and 25000), an initially perturbed state has been used to trigger this transition (figure $6 c$ ). For $G r=20000$, the initial perturbations are damped, and the instability threshold between the stationary convection and the oscillatory convection for $\mathrm{Pr}=0$ can be evaluated around $G r=22400$.

\subsubsection{Time characteristics}

The time signals corresponding to the oscillatory convection are perfectly periodic, with a main period $T_{2}$ (measured from the $L^{2}$ norm of the velocity components) decreasing from $T_{2}=0.173$ for $G r=25000$ to $T_{2}=0.121$ for $G r=45000$. These 

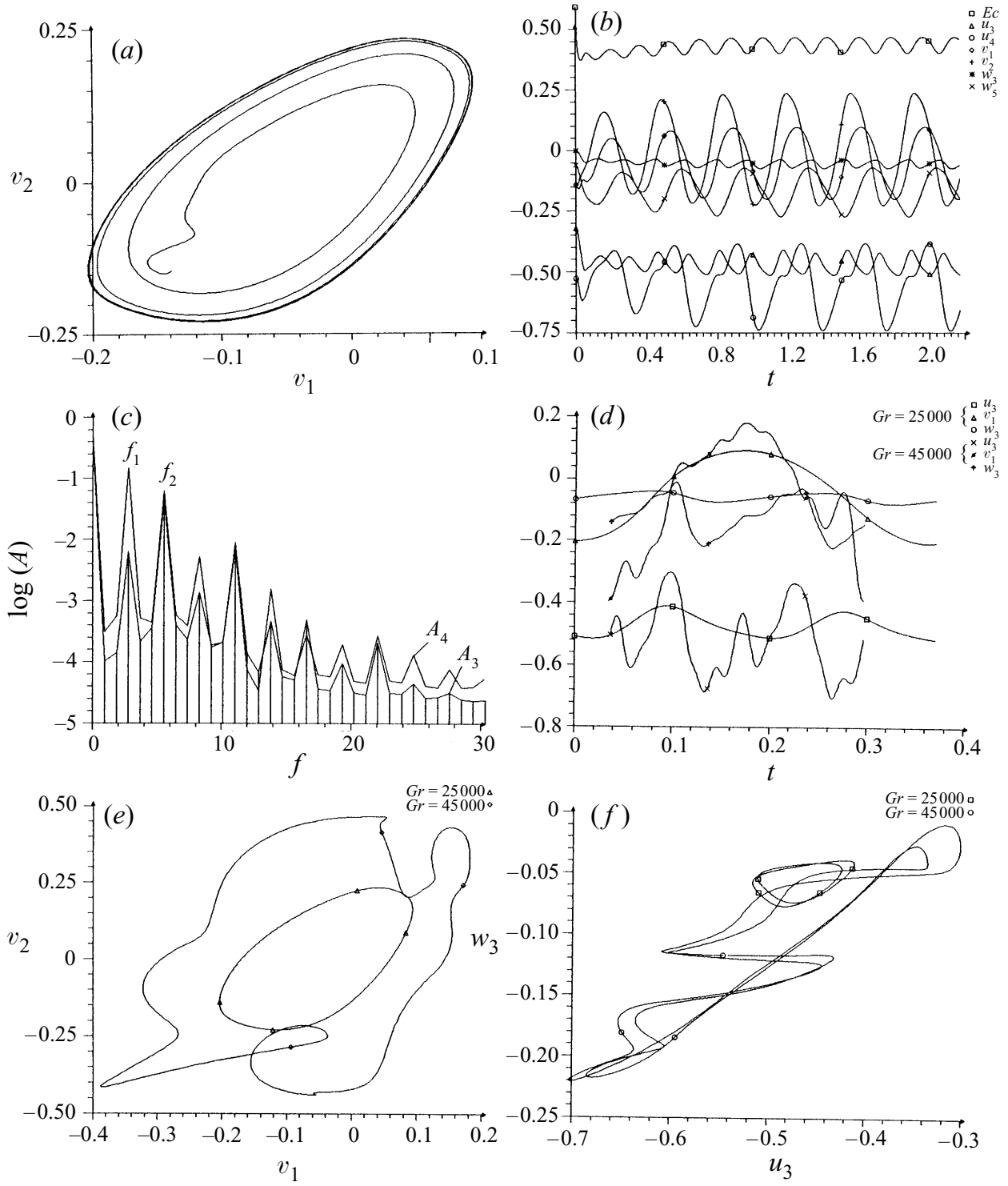

FIGURE 7. Time characteristics of the oscillatory convection in the $4 \times 2 \times 1$ cavity for $\mathrm{Pr}=0$ : $(a)$ phase trajectory $v_{2}=f\left(v_{1}\right)$ showing the initiation of the periodic flow at $G r=25000 ;(b)$ evolution with time of the velocity components at different points in the cavity and of the global kinetic energy $E c$ at $G r=25000$; signals with either a $T_{1}$ or a $T_{2}$ period are shown; $(c)$ corresponding power spectra of $u_{3}$ and $u_{4} ;(d)$ evolution with time of velocities over a period: comparisons between $G r=25000$ and $G r=45000 ;(e, f)$ comparisons between phase trajectories obtained at $G r=25000$ and $G r=45000\left((e) v_{2}=f\left(v_{1}\right) ;(f) w_{3}=f\left(u_{3}\right)\right)$. The subscripts $1-5$ refer to the points $P_{1}-P_{5}$ inside the cavity (see figure 1).

signals become more complex as $G r$ is increased: the Fourier power spectra given in figure $6(b, d)$ show a main peak at $f_{2}=1 / T_{2}$ for $G r=25000$, whereas several harmonics with similar intensity are present for $G r=45000$.

The time evolution of some local quantities, regarding the velocity components at some chosen locations (points $\left.P_{1}-P_{5}\right)$ is given in figure 7. Figure $7(a-c)$ concerns 

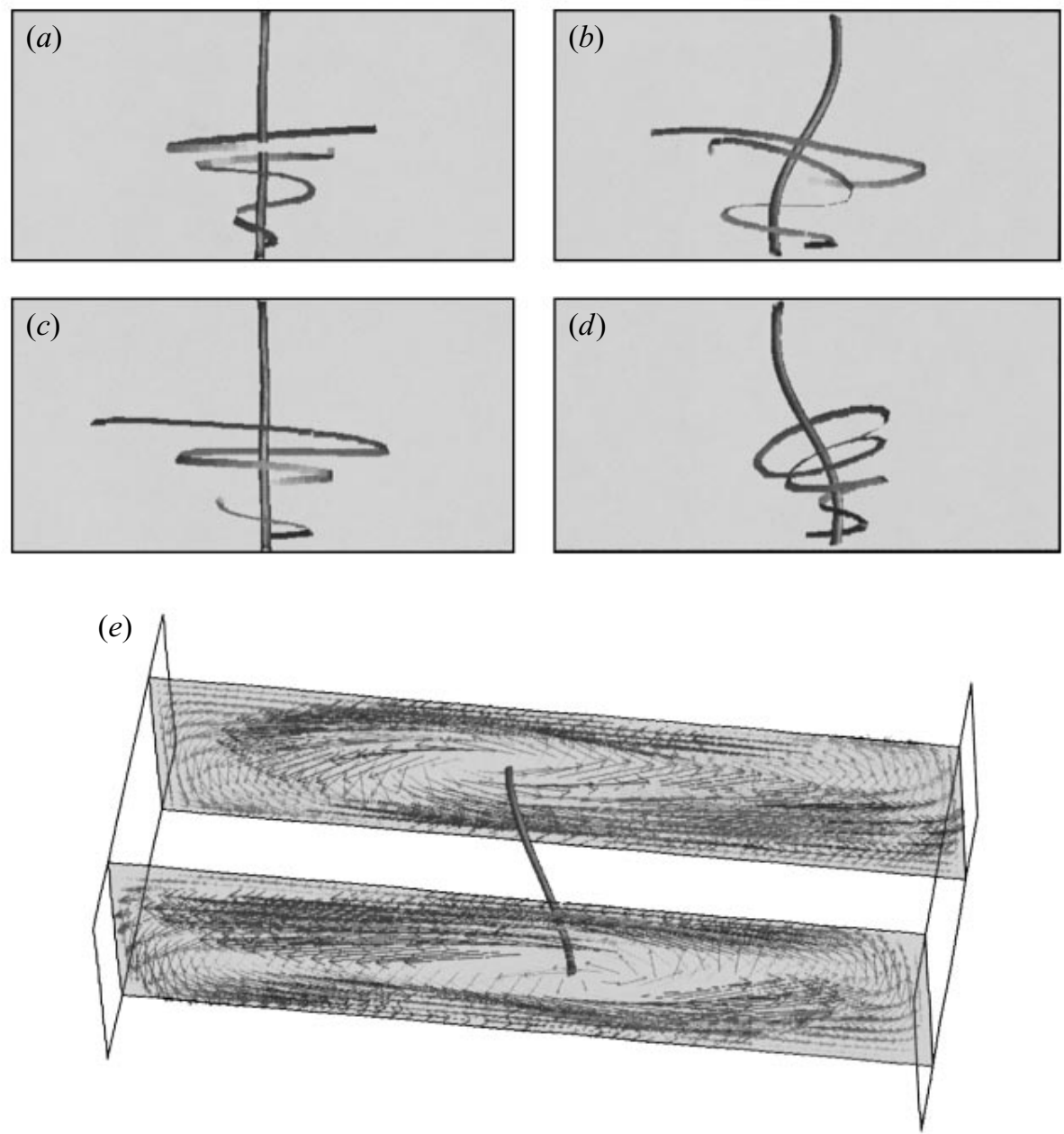

FiguRE 8. Oscillatory flow solution in the $4 \times 2 \times 1$ cavity for $\mathrm{Pr}=0$ and $\mathrm{Gr}=25000$ : $(a-d)$ top view of the streamline issued from the point $(2,0.1,0.35)$ and central vorticity tube passing through the centre point at four regularly spaced times during a period $T_{1}:(a) t=t_{0} ;(b) t=t_{0}+T_{1} / 4$; (c) $t=t_{0}+T_{1} / 2 ;(d) t=t_{0}+3 T_{1} / 4$. (e) Three-dimensional view of the velocity vector fields in two vertical planes parallel to $V_{l}$ and of the central vorticity tube featuring the centre of the roll at $t=t_{0}+3 T_{1} / 4$.

the case $G r=25000$. The phase trajectory $v_{2}=f\left(v_{1}\right)$ (figure $7 a$ ) shows clearly the setting up of the periodic flow initiated from the perturbed initial state. From the time evolutions given in figure $7(b)$, we can remark that when the points are outside the $V_{l}$ plane $\left(P_{1}, P_{4}\right.$ and $\left.P_{5}\right)$, the period of the signal is not equal to $T_{2}$, but to one twice as long $T_{1}=2 T_{2}\left(T_{1}=0.346\right.$ for $\left.G r=25000\right)$. This is confirmed by the power spectrum of $u_{4}$ (figure $7 c$ ) that has the main peak at the frequency $f_{1}$ which is half the main frequency $f_{2}$ obtained with the norms. On the other hand, when the points are situated in or near the $V_{l}$ plane (the original symmetry plane in the stationary state) $\left(P_{2}\right.$ and $\left.P_{3}\right)$, the velocity $v$ still presents a main peak at $f_{1}$, but the velocities $u$ and $w$ present a main peak at $f_{2}=2 f_{1}$ (see the power spectrum of $u_{3}$ in figure $7 c$ ). We will see in the next section that this result is related to symmetry properties of the oscillatory convection. This indicates also that the actual period of the global 

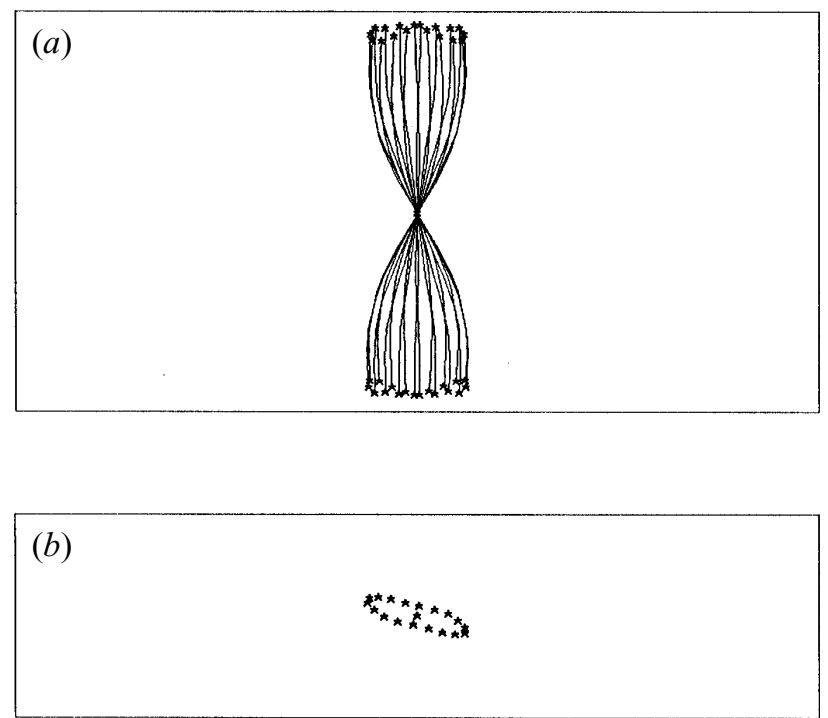

FiguRE 9. Oscillatory flow in the $4 \times 2 \times 1$ cavity for $\mathrm{Pr}=0$ and $\mathrm{Gr}=25000$. Vorticity lines (with an integration time of 0.36 ) passing through the central point of the cavity and corresponding to 18 equally spaced times during a period $T_{1}:(a)$ top view of the lines; $(b)$ front view of the extremities of the lines.

phenomenon is $T_{1}$ and not $T_{2}$. Figure $7(d-f)$ confirms by comparison between cases at $G r=25000$ and $G r=45000$ the increasing complexity of the oscillatory behaviour as $G r$ is increased.

\subsubsection{Spatial characteristics}

The spatial characterization of the oscillatory convection is performed for $G r=$ 25000 , one of the simplest cases treated for $\operatorname{Pr}=0$. The position of the central vorticity tube together with a characteristic streamline is given at four regularly spaced times during a period in figure $8(a-d)$ whereas the precise evolution of the central vorticity line during the whole period $T_{1}$ is given in figure 9. As shown in figure $8(e)$, these vorticity tubes and lines feature the successive positions of the axis of the central roll. In the stationary state, the axis of the roll was along the $y$-direction (see the vorticity tube in figure $5 c$ ) and $\Omega_{y}$ vorticity was thus generated. In the oscillatory state, the axis of the roll oscillates around its steady state position. This oscillation corresponds to a standing wave because of the symmetry point at the center of the cavity. Figure $9(b)$ shows that the roll oscillates mainly horizontally, corresponding to the creation of vertical vorticity $\Omega_{z}$, but also vertically corresponding to the creation of horizontal vorticity $\Omega_{x}$. The two oscillations are combined in such a way that the induced $\Omega_{z}$ and $\Omega_{x}$ vorticity have the same sign, and the evolution of the roll is perfectly continuous. The pictures $8(b-d)$, taken at $t_{0}+T_{1} / 4$ and $t_{0}+3 T_{1} / 4$ and which correspond to the maximum amplitude of the horizontal oscillation, indicate clearly the combined inclinations of the roll, corresponding to negative $\Omega_{z}$ and $\Omega_{x}$ vorticities in the first case and to positive vorticities in the second case. In the two other pictures $(8 a, c)$, the rolls are at the positions of minimum horizontal deviation.

A more careful examination of the results shows that the spatial oscillation of the roll corresponds to an elliptic precession with a fixed point at the centre of the cavity (this precession occurs in a direction opposite to the rotation of the flow in the roll, i.e. in the clockwise direction for figure $9 b$ ). The maximum horizontal 
and vertical deviations do not coincide exactly but are close one to another. The axis of the roll is quite strongly inclined in the core of the cavity, but it is more perpendicular to the lateral wall in the neighbourhood of this wall, giving there a kind of elliptic translation motion. The outer long-scale circulation seems less affected by the oscillatory convection: it looks like an adaptation to the oscillatory motion in the core. The flow along the horizontal walls remains roughly parallel to the $x$-direction, with only a small deviation of the flow (due to the vertical oscillation of the roll) from the part where the roll moves closer to that wall to the part where it moves away. Along the endwalls, the return motion to the lateral walls is no longer symmetric with respect to the $V_{l}$ plane but each of the return directions is successively favoured during the roll oscillation.

For $G r=45000$ the oscillatory convection still corresponds to the oscillations mentioned above, but the evolution with time is not as regular as for $G r=25000$. It presents some slight stagnation periods or reverse rotation sequences (see for example the phase trajectories of figure $7 e, f$ ). The spatial structure of the oscillatory flow is in fact more easily understood with some specific animations realized on Sun workstations (Henry \& Buffat 1990).

\subsection{Analysis of the symmetry properties}

The basic stationary state $\boldsymbol{U}_{0}$ obtained in the parallelepipedic cavity with rigid boundaries presented a reflection symmetry $S$ with respect to the $V_{l}$ plane:

$$
S \boldsymbol{U}_{0}=\boldsymbol{U}_{0}
$$

with by definition

$$
S:(x, y, z, t) \rightarrow(x, 2-y, z, t), \quad(U, V, W) \rightarrow(U,-V, W)
$$

and a reflection symmetry $S_{r}$ with respect to the $H_{t}$ axis:

$$
S_{r} \boldsymbol{U}_{0}=\boldsymbol{U}_{0}
$$

with by definition

$$
S_{r}:(x, y, z, t) \rightarrow(4-x, y, 1-z, t), \quad(U, V, W) \rightarrow(-U, V,-W) .
$$

These symmetries come from the invariances of the system restricted by the boundary conditions, and from specific properties of parity in the rigid case (see Laure 1987). The bifurcation to the oscillatory convection (corresponding to the oscillation of the roll) breaks these symmetries $S$ and $S_{r}$. It only preserves the symmetry $S_{c}$ with respect to the centre of the cavity $\left(S_{c}=S \cdot S_{r}\right)$. Because the oscillations of the roll occur successively on each side of its steady position, the $S$ and $S_{r}$ symmetries are maintained between states separated by half the period $T_{1} / 2=T_{2}$. Near the $V_{l}$ plane, the values of velocities $u$ and $w$ (and the temperature $T$ ) are then identical after half a period, whereas the velocity $v$ changes its sign. This accounts for the presence of a main peak at $f_{2}$ in the power spectrum of $u$ and $w$ (and $T$ ) near the $V_{l}$ plane, whereas the main peak in the power spectrum of $v$ is still at $f_{1}$. Outside the $V_{l}$ plane, the main frequency corresponds to $f_{1}$ for all the variables. These symmetry properties explain also why the $L^{2}$ norms of the velocities (i.e. the integral of the square of the velocity over the whole volume) have a period equal to $T_{2}=T_{1} / 2$ (figure $6 a$ ), leading to an effective main frequency at $f_{2}$ and to the suppression of the $f_{1}$ peak in the power spectrum (figure 6d). 


\subsubsection{General analysis}

These bifurcations with symmetry breaking can be analysed with the theory of dynamical systems (Iooss \& Joseph 1989; Iooss 1988). Although this analysis is now well known, we recall the main results. Near the bifurcation, the dynamics of our system is defined by an evolution equation of the form

$$
\frac{\partial U}{\partial t}=F(\mu, U(x, t))
$$

where $\mu$ is the control parameter (in our case $\mu=G r-G r_{c}$ and (5.3) is the NavierStokes equation (2.6)). For $\mu \leqslant 0$ there exists a stable solution $U_{0}(x, t)$, whereas for $\mu>0$ this solution is unstable to small disturbances and bifurcates to a new solution $U(x, t)$. We will now deduce some properties of the bifurcating solution $U(x, t)$ from the symmetry properties of the basic state $U_{0}(x, t)$.

Let us consider the case of a stationary basic state $U_{0}(x)$ (i.e. satisfying $F\left(\mu, U_{0}\right)=0$ ) which bifurcates to a periodic state $U(x, t)$ through a Hopf bifurcation at $\mu=0$, and assume that $U_{0}$ has the symmetry $S$ which commutes with $F$ :

$$
S U_{0}=U_{0} \text { and } F\left(\mu, S U_{0}\right)=S F\left(\mu, U_{0}\right) .
$$

The linear operator $L$, associated with the linearization of the equation (5.3) around $U_{0}$ is defined by

$$
L(\mu)=\frac{\partial F\left(\mu, U_{0}\right)}{\partial U}
$$

and commutes with $S$. Let us assume now that $\xi$ and $\bar{\xi}$ are the two complex conjugate eigenvectors associated with the two most unstable eigenvalues $\sigma(\mu)=\eta(\mu)+\mathrm{i} \omega(\mu)$ and $\bar{\sigma}=\eta(\mu)-\mathrm{i} \omega(\mu)$ of the linear operator $L(\mu)$ :

$$
L(\mu) \xi=\sigma(\mu) \xi \text { with } \eta(\mu)<0 \text { for } \mu<0 \text { and } \eta(\mu) \geqslant 0 \text { for } \mu \geqslant 0 .
$$

As $L$ commutes with $S, S \xi$ is also an eigenvector of $L$ associated with the same eigenvalue $\sigma$, so that $S \xi$ is carried by $\xi$ : $S \xi=\alpha \xi$. As the symmetry $S$ verifies $S^{2}=I d$ ( $I d$ is the identity operator), we obtain $\alpha^{2}=1$, i.e.

$$
S \xi= \pm \xi \text {. }
$$

Near the threshold, the bifurcating solution $U(x, t)$ is a combination of the basic state $U_{0}$ and a fluctuation $u(x, t)$ spanned mainly by $\xi$ and $\bar{\xi}$ (Iooss \& Joseph 1989):

$$
u(x, t)=z(t) \xi+\bar{z}(t) \bar{\xi}+w(x, t) \text { with } w=O\left(|z|^{2}\right) .
$$

Using the time invariance of the solution, the amplitude equation for $z$ may be written as

$$
\frac{\mathrm{d} z}{\mathrm{~d} t}=(a \mu+\mathrm{i} \omega) z-b|z|^{2} z .
$$

This is the normal form of the Hopf bifurcation, where the first term accounts for the linearized operator $L$ (with $\sigma=a \mu+\mathrm{i} \omega$ ), and the second term accounts for the nonlinear saturation of the linear perturbation. This equation has a stable solution for $\mu \geqslant 0$ :

$$
z(t)=\alpha \mu^{1 / 2} \mathrm{e}^{\mathrm{i} \omega t} \text { with } \alpha^{2}=\frac{a}{b}
$$

which is periodic with a period $T=2 \pi / \omega$ and has an amplitude proportional to the square root of the control parameter $\mu$. The bifurcating solution is then

$$
U(x, t)=U_{0}+\alpha \mu^{1 / 2} \mathrm{e}^{\mathrm{i} \omega t} \boldsymbol{\xi}(x)+\alpha \mu^{1 / 2} \mathrm{e}^{-\mathrm{i} \omega t} \bar{\xi}(x) .
$$




$\begin{array}{ccc}\text { Mode } & \text { Eigenvalues } & \text { \% energy } \\ 1 & 0.7682 & 76.82 \\ 2 & 0.1980 & 96.62 \\ 3 & 0.0218 & 98.80 \\ 4 & 0.0090 & 99.70\end{array}$

TABLE 1. Normalized eigenvalues of the POD modes and their cumulative contribution to the total energy for $\mathrm{Gr}=25000$ and $\mathrm{Pr}=0$.

Applying the symmetry operator $S$ on the bifurcating solution leads to

$$
S U(x, t)=U_{0}+\alpha \mu^{1 / 2} \mathrm{e}^{\mathrm{i} \omega t} S \xi(x)+\alpha \mu^{1 / 2} \mathrm{e}^{-\mathrm{i} \omega t} S \bar{\xi}(x) \text { with } S \xi= \pm \xi .
$$

- If the eigenvector $\xi$ is invariant under $S$ (i.e. $S \xi=\xi$ ), then the bifurcating oscillatory solution is pointwise invariant under $S$ :

$$
S U(x, t)=U(x, t) .
$$

In this first case, the Hopf bifurcation occurs without symmetry breaking. That corresponds to the first oscillatory bifurcation observed in the two-dimensional cavity.

- If the eigenvector $\xi$ is such that $S \xi=-\xi$, the $S$ symmetry is broken but the bifurcating solution verifies

$$
\begin{aligned}
S U(x, t) & =U_{0}+\alpha \mu^{1 / 2} \mathrm{e}^{\mathrm{i} \omega(t+\pi / \omega)} \boldsymbol{\xi}(x)+\alpha \mu^{1 / 2} \mathrm{e}^{-\mathrm{i} \omega(t+\pi / \omega)} \bar{\xi}(x) \\
& =U(x, t+\pi / \omega) .
\end{aligned}
$$

Thus, a Hopf bifurcation with a symmetry breaking is characterized by an antisymmetric perturbation, and the bifurcating solution preserves the symmetry between states separated by half a period.

\subsubsection{Three-dimensional oscillatory bifurcation}

In the three-dimensional case, the first bifurcation from the stationary state $\boldsymbol{U}_{0}(x, y, z)$ to an oscillatory convection (with a period $\left.T_{1}=2 T_{2}\right)$ is a Hopf bifurcation with symmetry breaking on $S$ and $S_{r}$. From the above analysis, the bifurcating solution $\boldsymbol{U}(x, t)$ has the following symmetry properties:

$$
\boldsymbol{S} \boldsymbol{U}(x, y, z, t)=\boldsymbol{U}\left(x, y, z, t+T_{2}\right), \quad S_{r} \boldsymbol{U}(x, y, z, t)=\boldsymbol{U}\left(x, y, z, t+T_{2}\right) .
$$

And as $S_{c}=S \cdot S_{r}$, the bifurcating solution is also pointwise invariant under $S_{c}$ :

$$
S_{c} \boldsymbol{U}(x, y, z, t)=\boldsymbol{U}(x, y, z, t) .
$$

From our data, it was not possible to have $\boldsymbol{U}_{0}(x, y, z)$, the steady flow at the oscillatory threshold and, for $G r=25000$, to deduce the perturbation as expressed by (5.11). However, by averaging the numerical results over the period, we have obtained the mean flow $\langle\boldsymbol{U}\rangle_{t}(x, y, z)$ and deduced the fluctuating flow $\boldsymbol{u}(x, y, z, t)=$ $\boldsymbol{U}(x, y, z, t)-\langle\boldsymbol{U}\rangle_{t}(x, y, z)$. This fluctuating flow has been analysed by the method of proper orthogonal decomposition (POD), namely the method of snapshots as used by Deane et al. (1991) and discussed by Sirovich \& Park (1990). For $G r=25000,181$ snapshots regularly spaced during the period $T_{1}$ have been used for the decomposition of the fluctuating flow. The four largest eigenvalues corresponding to the most energetic modes are shown in table 1 . We observe a quick decrease of the contributions of the modes, and clearly the first mode dominates, comprising more than $76 \%$ of the 
(a)

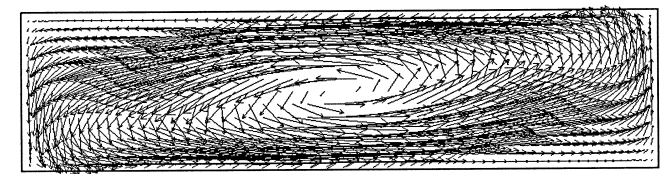

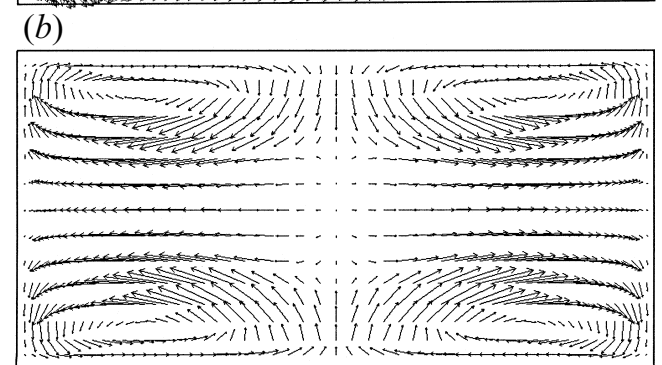

(c)

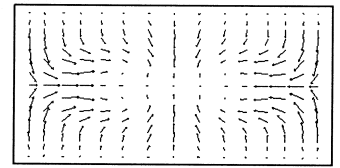

$(d)$

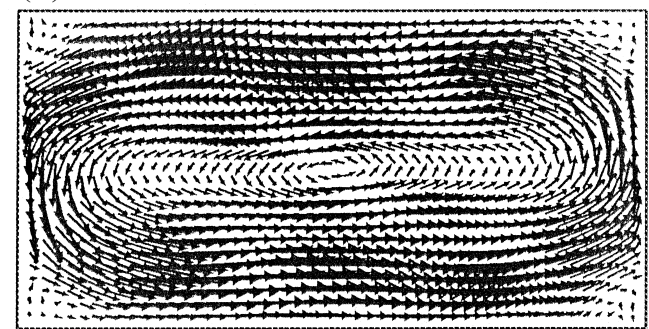

(e)

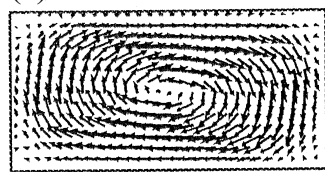

FiguRE 10. Oscillatory flow in the $4 \times 2 \times 1$ cavity for $P r=0$ and $G r=25000$ : velocity field in the principal middle planes, for the mean flow ( $a, V_{l}$ plane; $b, H_{l}$ plane; $c, V_{t}$ plane) and for the dominant first mode $\left(d, H_{l}\right.$ plane; $e, V_{t}$ plane).

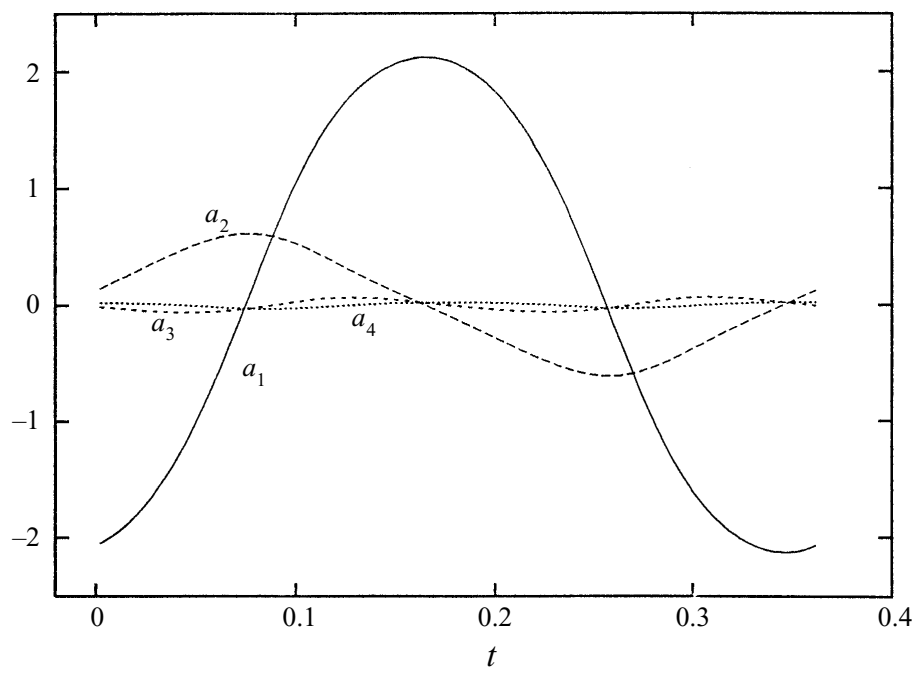

FiguRE 11. Time variation of the amplitudes of the first four modes.

'energy' of the motion. The spatial structure of the mean flow and of the first mode is shown in figure 10. The mean flow compares well with the steady flow solution at $G r=20000$ ( $S$ and $S_{r}$ symmetries, concentrated roll in the $V_{l}$ plane, four vortices in the $H_{l}$ plane). As expected from $\S 5.3 .1$, the dominant first mode is anti-symmetric with respect to $S$ and $S_{r}$, with no flow in the $V_{l}$ plane. In the horizontal plane $H_{l}$ (figure $10 d$ ), the flow for this first mode corresponds mainly to a rotation around the centre of the cavity, which creates the vorticity $\Omega_{z}$ responsible for the horizontal 


$\begin{array}{ccccc}t & t_{0} & t_{0}+T_{1} / 4 & t_{0}+T_{1} / 2 & t_{0}+3 T_{1} / 4 \\ \frac{1}{2}\left\langle|u|^{2}\right\rangle & 0.02315 & 0.01788 & 0.02338 & 0.01755\end{array}$

TABLE 2. Average kinetic energy of the fluctuation $\boldsymbol{u}$ for $G r=25000$ and $P r=0$ at four equally spaced times during a period $T_{1}$. The average kinetic energy of the mean flow $\boldsymbol{U}_{0}$ is 0.24680 .

oscillation of the main roll. In the vertical plane $V_{t}$ (figure $10 e$ ) we observe also a rotation, which creates the vorticity $\Omega_{x}$ responsible for the vertical oscillation of the main roll. The second mode looks similar to the first, with the same symmetries and similar rotations in the $H_{l}$ and $V_{t}$ planes (they together contain more than $96 \%$ of the 'energy'). It is only with the third and fourth modes (only 3\% of the 'energy') that flow structures with the same symmetries as the mean flow are found. As regards temporal response of the modes (figure 11), the third and fourth modes oscillate with twice the frequency of the first two modes, a phase shift of about $\pi / 2$ being observed between the first and second modes.

\subsection{Energy budget}

The average kinetic energy of the perturbation of the periodic flow $0.5\left\langle\boldsymbol{U}^{2}\right\rangle$ is given in table 2 at four different times during a period for $\mathrm{Gr}=25000$ and $\mathrm{Pr}=0$. These variations are around $10 \%$ of the average kinetic of the mean flow, $0.5\left\langle\langle\boldsymbol{U}\rangle_{t}^{2}\right\rangle$. The spatial localization of the kinetic energy is given in figure $12(a-d)$, where the kinetic energy of the fluctuation at a given time in the period is compared with the kinetic energy of the mean flow. The maxima of the kinetic energy are located in the middle of the cavity within two zones. For the mean flow, these two zones are along the two horizontal walls of the cavity on each side of the $H_{l}$ plane, whereas for the perturbation, they are near the two vertical lateral walls on each side of the $V_{l}$ plane.

If we write now the equation of conservation for the fluctuating kinetic energy and calculate the different contributions, it is found for $G r=25000$ and $P r=0$ that the production of fluctuating kinetic energy comes mainly from the shear of the mean flow, with a main term corresponding to $w^{\prime}\left(\partial u_{0} / \partial z\right) u^{\prime}$. The zones of production due to this dominant contribution are located near the lateral walls within the domain of strong fluctuating kinetic energy presented in figure $12(c, d)$.

\subsection{Influence of $\mathrm{Pr}$}

\subsubsection{General results}

Experimental results are available for such rigid cavities $(4 \times 2 \times 1)$ with adiabatic lateral walls in the case of mercury which corresponds to $\operatorname{Pr}=0.026$ (Hart \& Pratte 1990; Pratte \& Hart 1990; Hung \& Andereck 1990). In order to allow some comparisons, simulations have been done for $\operatorname{Pr} \neq 0$ in the adiabatic case, mainly for $P r=0.026$ (from $G r=30000$ to $G r=45000$ ).

In cases with small but non-zero $\operatorname{Pr}(\operatorname{Pr}=0.015$ and $\operatorname{Pr}=0.026)$, beyond a certain threshold an oscillatory behaviour globally of the same type as for $P r=0$ is obtained. The time evolution of the norm of $v$ is given in figure 13 for the three cases, $\operatorname{Pr}=0$ at $G r=45000$ and $G r=25000$, and $P r=0.026$ at $G r=45000$. The main result is the strong increase of the frequency when $P r$ is different from zero. For example, as can be seen in table 3, for $G r=45000$ the frequency changes from 4.1 for $P r=0$, to 14.8 for $\operatorname{Pr}=0.015$ and to 16.0 for $P r=0.026$. For situations closer to the thresholds $(G r=25000$ for $P r=0$ and $G r=35000$ for $P r=0.026)$ the frequency increases 


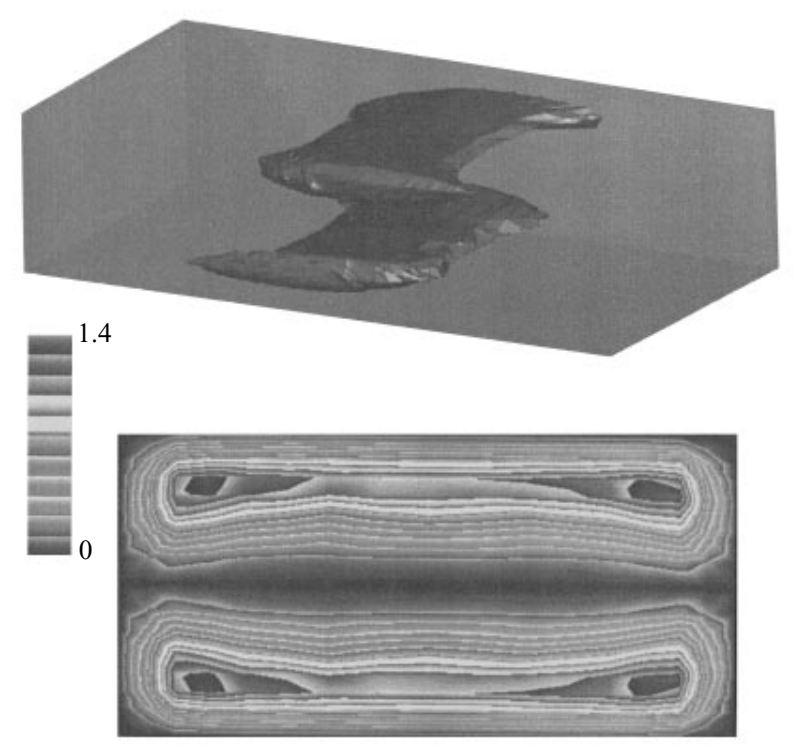

(a)

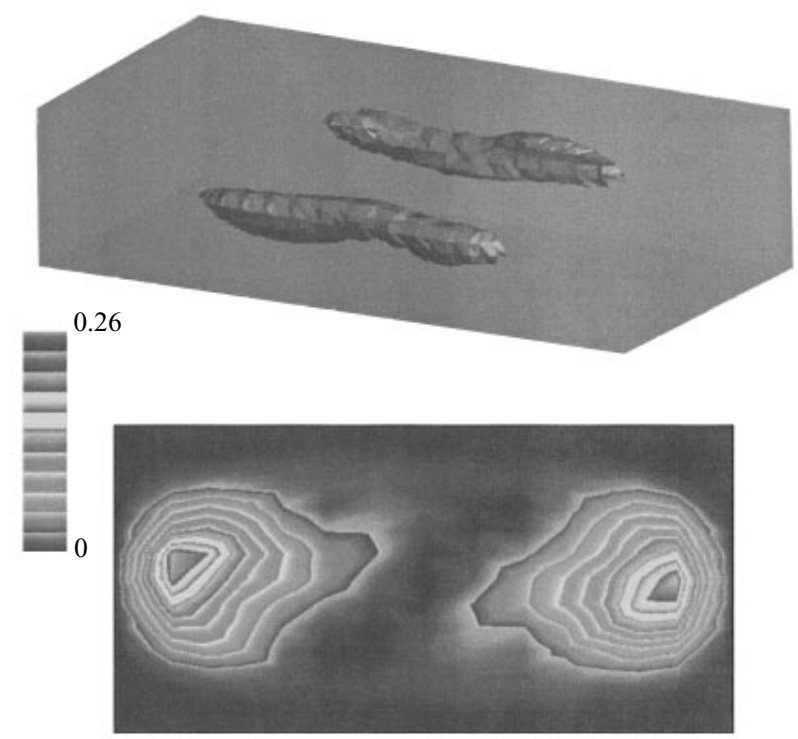

(c)

FIGURE 12. Oscillatory flow in the $4 \times 2 \times 1$ cavity for $P r=0$ and $G r=25000$. Comparison between the kinetic energy field of the mean flow and of the perturbation at a given time in the period: $(a)$ three-dimensional view of the mean flow kinetic energy iso-surface corresponding to half of the maximum value; $(b)$ mean flow kinetic energy in the transverse vertical plane $V_{t} ;(c)$ three-dimensional view of the fluctuation kinetic energy iso-surface corresponding to half of the maximum value; $(d)$ fluctuation kinetic energy in the transverse vertical plane $V_{t}$.

from $2.9(P r=0)$ to $13.8(P r=0.026)$. The situations at $P r=0$ and $P r=0.026$ are compared through time variations (figure $14 a$ for $P r=0.026$ and figure $7 b$ for $P r=0$ ) and phase diagrams (figure $14 b, c$ ). The two cases $G r=45000$ at $P r=0.026$ and $G r=25000$ at $P r=0$ give the same type of evolution with a similar intensity. Smaller fluctuations are obtained for $\mathrm{Pr}=0.026$ in situations closer to the threshold 


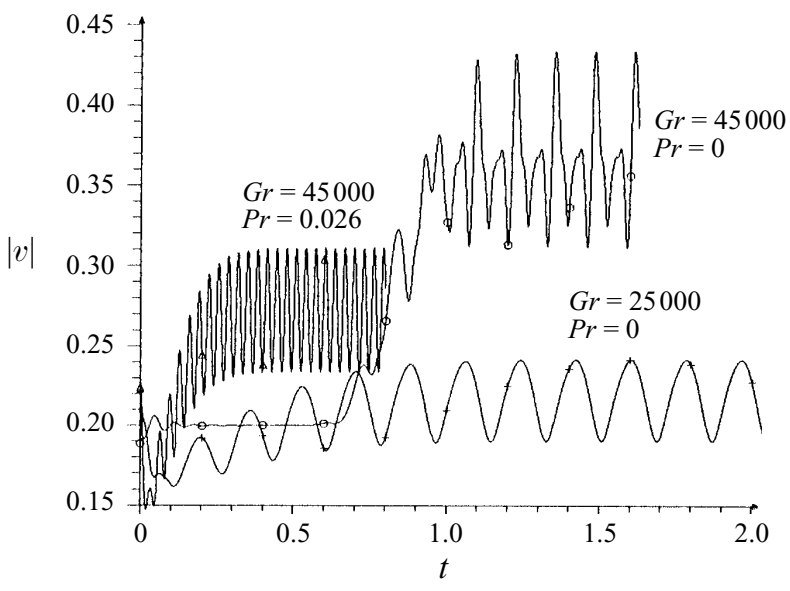

FIGURE 13 . Time characteristics of the oscillatory convection in the $4 \times 2 \times 1$ cavity. Comparison of the time evolution of the $L^{2}$ norm of $v$ for $P r=0$ at $G r=25000$ and $G r=45000$, and for $\operatorname{Pr}=0.026$ at $G r=45000$.

$\begin{array}{cccc}G r \backslash P r & 0 & 0.015 & 0.026 \\ 25000 & 2.9 & & \\ 35000 & & & 13.8 \\ 45000 & 4.1 & 14.8 & 16.0\end{array}$

TABLE 3. Frequency $f_{1}$ of the time-dependent flow occurring in the $4 \times 2 \times 1$ rigid cavity for small values of $\mathrm{Pr}$ and different values of $\mathrm{Gr}$.

as at $G r=35000$ (figure $14 b, c$ ), indicating that the growth of the perturbation with $G r$ at $P r=0.026$ seems to be slower than at $P r=0$.

The oscillation of the roll is depicted in figure 15 for $G r=40000$ at $P r=0.026$ : the elliptic precession of the central vorticity line obtained at $P r=0$ (figure 9) appears rather as an oscillating motion along a straight line corresponding to the large axis of the previous ellipse, and this motion, as indicated by the stronger frequency, occurs more quickly. Evolution with time of the temperature field can be observed in figure 16 where the isotherms on the top and side boundaries are given at four instants throughout the period: it can be noticed that the deformations of the temperature field follow the oscillations of the velocity field.

For $\operatorname{Pr}=0.026$ (experimental case), the critical Grashof number (assuming a Hopf bifurcation and using extrapolation to zero amplitude) has been found at $G r_{c}=33700 \pm 300$ with a critical frequency $f_{1 c}=13.6$ obtained by extrapolation of the period to $G r_{c}$.

\subsubsection{Comparisons with experiments}

The available experimental results (given in the publications mentioned above) correspond to the time series of the temperature at some particular locations and to the corresponding power spectra. In Hung \& Andereck (1990), the temperature is measured at a single point at the top centre of the cavity $(x=2, y=1, z=1)$. The critical Grashof number $G r_{c}$ for the onset of oscillations is estimated around $G r_{c}=38870$. Just beyond $G r_{c}$, they observe a very steady oscillation with a primary frequency $f_{2}=27.1$, followed soon by the first subharmonic at $f_{2} / 2$. When increasing 
(a)

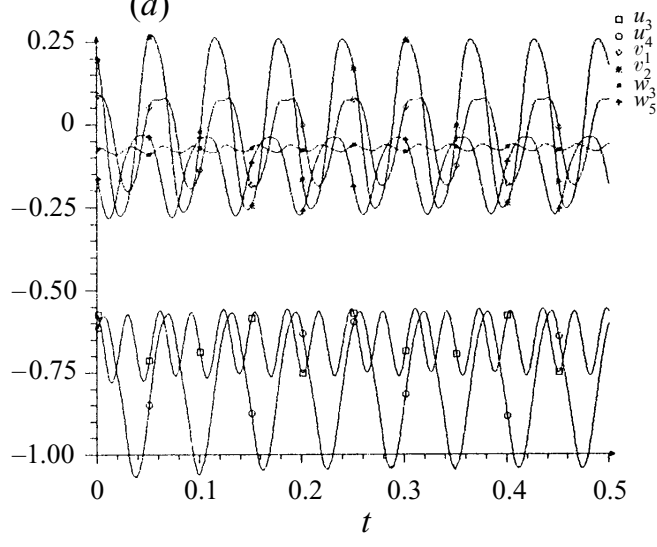

(c)

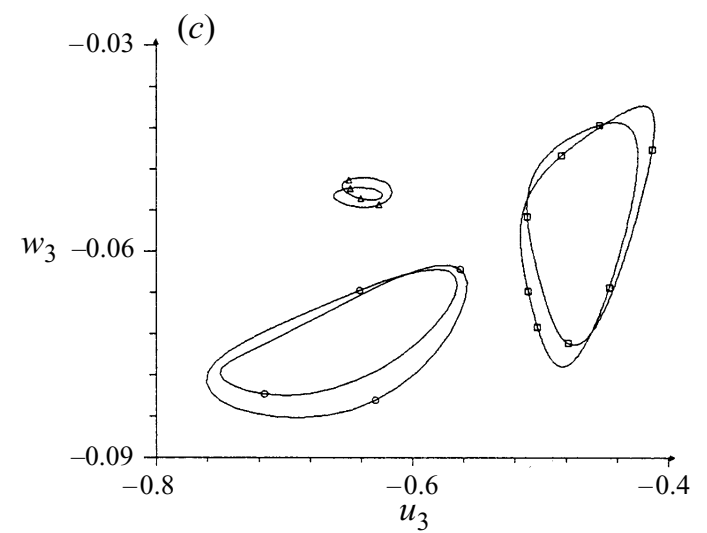

(b)

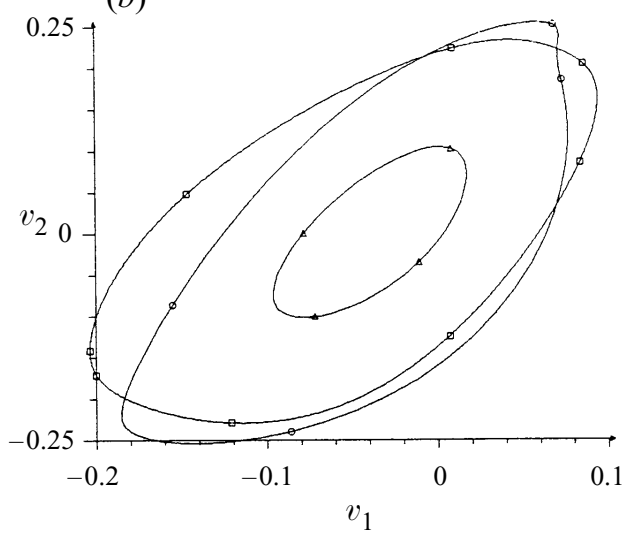

0.1

FIGURE 14. Time characteristics of the oscillatory convection in the $4 \times 2 \times 1$ cavity for $\mathrm{Pr}=0.0026$ : (a) evolution with time of the velocities at different points in the cavity for $G r=45000 ;(b, c)$ comparisons between phase trajectories obtained at $\operatorname{Pr}=0$ and at $\operatorname{Pr}=0.026:(b) v_{2}=f\left(v_{1}\right)$, (c) $w_{3}=f\left(u_{3}\right)$. $(\square, P r=0$ for $G r=25000 ; \triangle, P r=0.026$ for $G r=35000 ; \bigcirc, P r=0.026$ for $G r=45000)$.

the Grashof number up to 132600 , they obtain the successive appearance of the subharmonics $f_{2} / 3, f_{2} / 6, f_{2} / 9$ and $f_{2} / 18$. In Hart \& Pratte (1990), the temperature is measured in the $V_{t}$ plane $(x=2)$ near the top at different $y$ and different depths. The value of $G r_{c}$ is given there around $G r_{c}=42300$. The flow starts to oscillate at $f_{1}=14.3$, but it is mentioned that the centre probe (at $\left.y=1\right)$ presents a spectrum that rapidly becomes dominated by its second harmonic $2 f_{1}$, while the off-centre probes at $y=0.25$ and $y=0.75$ keep the lower frequency $f_{1}$. Further evolutions correspond to period doubling $\left(f_{1} / 2\right.$ and $\left.f_{1} / 4\right)$ and to other more complicated subharmonics. In Pratte \& Hart (1990), the authors indicate that they have realized some measurements outside the $V_{t}$ plane and they give for the same case a smaller critical Grashof number, $G r_{c}=39600$. They do not give the new value for the fundamental frequency $f_{1}$, but mention a further evolution corresponding to the subharmonic $5 f_{1} / 8$.

These experimental results compare well with our numerical results, specially on the following points:

- The critical Grashof number given by the simulation, $G r_{c}=33700$, is a little smaller than the values given by the experiments. This discrepancy may be attributed to numerical errors because our mesh is a little too coarse in some parts of the 


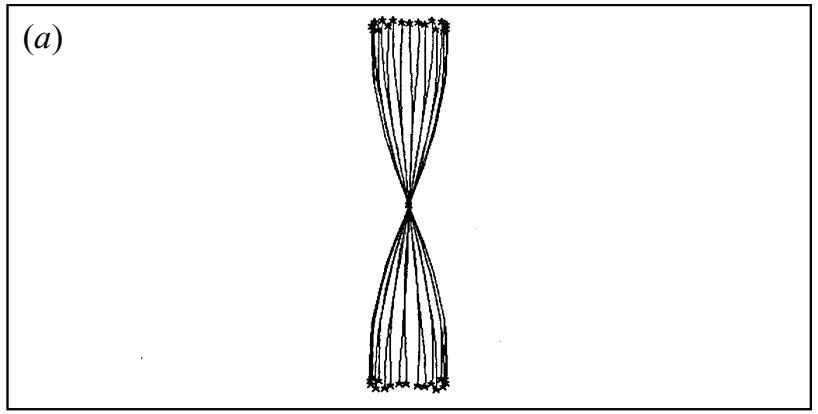

(b)

FiguRE 15 . Oscillatory flow in the $4 \times 2 \times 1$ cavity for $P r=0.026$ and $G r=40000$. Vorticity lines (with an integration time of 0.38 ) passing through the central point of the cavity and corresponding to 17 regularly spaced times during a period $T_{1}:(a)$ top view of the lines; $(b)$ front view of the extremities of the lines.

(a)

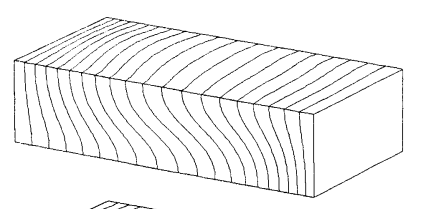

(b)

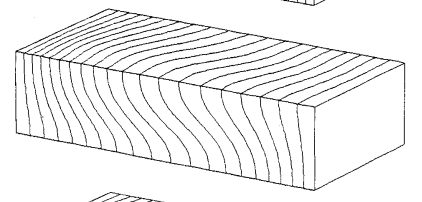

(c)

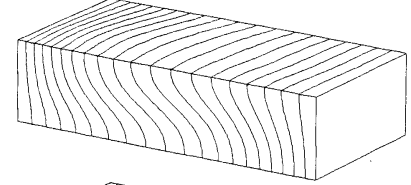

(d)

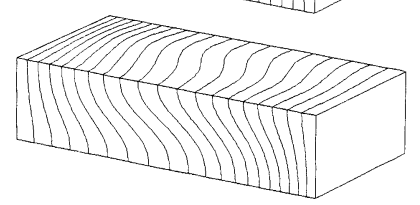

FIGURE 16. Temperature field in the $4 \times 2 \times 1$ cavity for $P r=0.026$ and $G r=40000$. Isotherms on the walls given at four equally spaced times during a period $T_{1}$ : (a) $t=t_{0} ;(b) t=t_{0}+T_{1} / 4 ;(c)$ $t=t_{0}+T_{1} / 2 ;(d) t=t_{0}+3 T_{1} / 4$.

cell, but also to experimental uncertainties because finite-amplitude oscillations are required to detect the threshold during the experiments. Concerning experimental uncertainties, Hung \& Andereck (1990) specify that the temperature difference $\Delta T$ at the critical threshold corresponds to $2.24^{\circ} \mathrm{C}$, with a temperature regulation of about $\pm 0.02{ }^{\circ} \mathrm{C}$ which is much smaller than the oscillation amplitude observed at the 

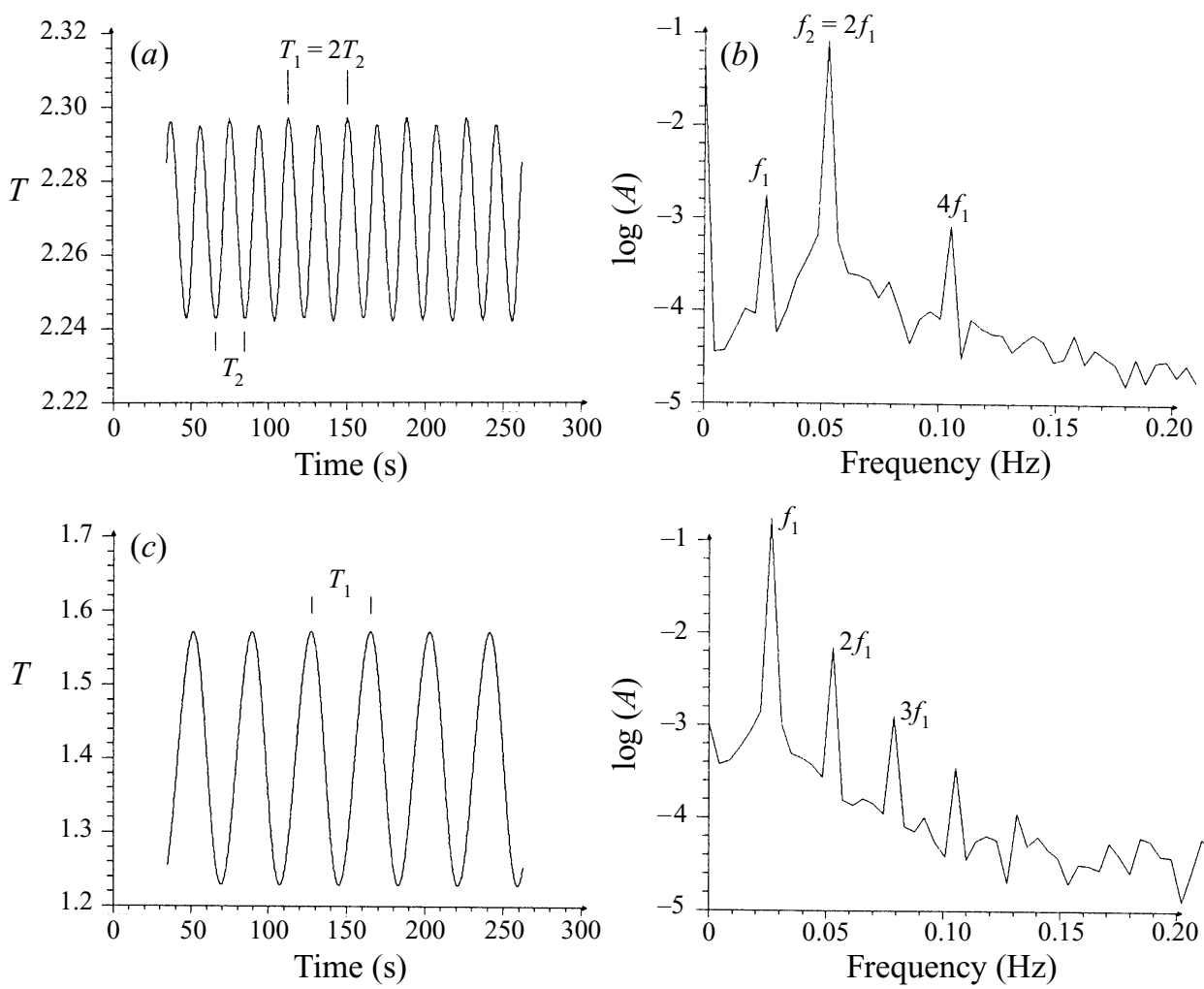

FIGURE 17. Time series of the temperature and corresponding power spectra for $\operatorname{Pr}=0.026$ and $G r=40000:(a)$ time series of the temperature at point $P_{2}$ (in the $V_{l}$ plane); $(b)$ corresponding power spectrum with a main frequency $f_{2}=2 f_{1} ;(c)$ time series of the temperature at point $P_{4}$ (outside the $V_{l}$ plane); $(d)$ corresponding power spectrum with a main frequency $f_{1}$.

onset, and that this temperature difference is increased by steps of $0.12{ }^{\circ} \mathrm{C}$. Thus the measurements by steps of $0.12^{\circ} \mathrm{C}$ compared to $2.24^{\circ} \mathrm{C}$ give an uncertainty on the critical $\mathrm{Gr}$ of about 2000. Moreover, according to the simulations, the amplitude of the temperature oscillations at the top centre of the cavity where Hung \& Andereck (1990) made their measurements (for example at the point $P_{2}$ ) is about $0.01,0.03$ and 0.06 (non-dimensional values) for $G r=35000,38000$ and 40000 respectively, which corresponds to $0.005,0.017$ and $0.028^{\circ} \mathrm{C}$ in the experimental situation. When compared to the regulation precision of $0.02^{\circ} \mathrm{C}$, this leads to a possible overestimation of the critical threshold during the experiment, of up to 5000 .

- The values of the critical frequencies are in good agreement. The global oscillation of the roll observed by the simulations corresponds to a main frequency $f_{1}=13.6$, but because of the symmetry properties of the evolution $(S$ symmetry for two states separated by $T_{1} / 2$ ) the frequency $f_{2}=2 f_{1}=27.2$ is also observed near the $V_{l}$ plane. The values of these frequencies agree well with the experimental observations which confirm also our interpretation of the motion: Hung \& Andereck (1990) made measurements in the $V_{l}$ plane (top centre) and obtained the two harmonics $f_{2}=27.1$ and $f_{2} / 2$; Hart \& Pratte (1990) generally obtained the harmonic $f_{1}=14.3$, but the centre probe also gave the harmonic $2 f_{1}=f_{2}=28.6$.

- Some comparisons can be made on the evolution of the temperature field. We give in figure 17 for the case $G r=45000$ at $P r=0.026$ the evolution with time of the 
(a)

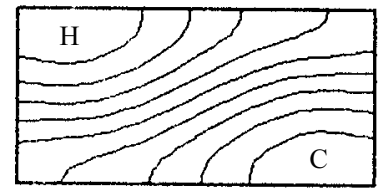

(b)

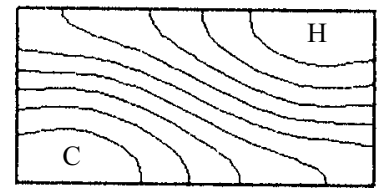

FIGURE 18. Temperature field in the $4 \times 2 \times 1$ cavity for $P r=0.026$ and $G r=40000$. Isotherms in the $V_{t}$ plane given at two times $t_{0}$ and $t_{0}+T_{1} / 2$. The letters $\mathrm{H}$ and $\mathrm{C}$ indicate respectively the hot and cold zones.

temperature at the two points $P_{2}$ and $P_{4}$, and the corresponding power spectra. The evolution at $P_{2}$ (which is located in the $V_{l}$ plane just below the top centre) presents an apparent period of $T_{2}$ and corresponds to a main peak at $f_{2}$ with a small contribution at $f_{1}$ : it can be compared with a good similarity with figures $1 \mathrm{~b}$ and $1 \mathrm{~b}^{\prime}$ of Hung \& Andereck (1990), and qualitatively with figure 3a of Hart \& Pratte (1990). The evolution at $P_{4}$ (which is located outside the $V_{l}$ plane) presents an apparent period of $T_{1}$ and corresponds to a main peak at $f_{1}$ with smaller subharmonics contributions: it can be qualitatively compared with figure 3b of Hart \& Pratte (1990). Finally, the isotherms obtained on the top boundary and at times corresponding to the maximum deformation $\left(t=t_{0}+T / 4\right.$ and $\left.t=t_{0}+3 T / 4\right)$ (figure 16b,d) compare qualitatively well with those given in figure 5 by Pratte \& Hart (1990).

- Concerning the characterization of the next bifurcations, there is no perfect agreement between the experimental studies: Hung \& Andereck (1990) found a subharmonic at $f_{2} / 3=0.66 f_{1}$, Hart \& Pratte (1990) a period doubling at $f_{1} / 2=0.5 f_{1}$ and Pratte \& Hart (1990) a subharmonic at $5 f_{1} / 8=0.63 f_{1}$. In our case, an increase of $G r$ leads to a period doubling at $f_{1} / 2$ for $G r=150000$ (Buffat \& Henry 1991). The frequency $f_{1} / 2$ which indicates a period corresponding to two oscillations of the roll can be expected as it would only mean that two successive oscillations of the roll are now a little different.

\subsubsection{Discussion}

We have seen that the increase of $\operatorname{Pr}$ leads to an increase of the critical $G r$ for oscillatory convection and to a strong increase of the frequency. A value of $\operatorname{Pr}$ different from zero leads to longitudinal deformations of the isotherms (observable in the $V_{l}$ plane) which decrease the longitudinal temperature gradient in the core of the cavity responsible for the intensity of the motion. As the transition to the instability has a dynamical origin, an increase of $G r$ will then be necessary to reach a critical velocity or a critical vorticity $\Omega_{y}$.

The oscillatory convection, corresponding to spatial oscillations of the roll, leads for $P r \neq 0$ to transversal deformations of the isotherms (well perceptible in the $V_{t}$ plane, see figure 18) which tend to stabilize the oscillation of the roll. For $\operatorname{Pr}=0$ the stabilization of the roll oscillation depends only on dynamical effects (pressure and viscosity), while for $\operatorname{Pr} \neq 0$ it is strongly affected by large thermal stabilization effects, which could explain the strong decrease of the period when $\mathrm{Pr}$ is different from zero.

For these cases with $P r \neq 0$, the principal characteristics of the kinetic energy transfer from the mean flow are still found to be like those for $P r=0$, with a dominant contribution coming from $w^{\prime}\left(\partial u_{0} / \partial z\right) u^{\prime}$. The extra term corresponding to the contribution of the fluctuating temperature in buoyancy is an order of magnitude smaller. Moreover all the terms of the equation of conservation of the fluctuating thermal energy are globally smaller. 


\section{General discussion and comparisons}

\subsection{Three-dimensional oscillatory instability}

The oscillatory instability found in the three-dimensional case is of pure dynamical origin. It is obtained even in the limit $P r=0$ corresponding to a temperature field completely frozen in its diffusive equilibrium and for which no temperature fluctuations are allowed. A small value of $\mathrm{Pr}$ modifies the characteristics of the instability but does not change its fundamental nature.

This oscillatory instability corresponds to a global oscillation of the roll with a fixed point at the centre of the cavity, appearing as a standing wave. It looks very different from the oscillatory longitudinal rolls instability which is predicted by the stability analysis of the Hadley circulation for small Prandtl numbers (Laure 1987; Wang \& Korpela 1989) and which has a thermal origin as the threshold increases strongly for $\mathrm{Pr} \rightarrow 0$. However, quite similar oscillations of rolls are predicted by theoretical analysis in different situations with small-Pr fluids (Clever \& Busse 1974; Nagata \& Busse 1983; Meneguzzi et al. 1987).

These situations correspond to extended fluid layers heated from below where a steady convection in the form of two-dimensional counter-rotating rolls is obtained. For small-Pr fluids, these rolls can be destabilized through an oscillatory instability which corresponds to a global oscillation of the rolls propagating in time along the roll axis (travelling wave). The frequencies of the oscillations $(13<f<19$ for $P r=0.025$ (Clever \& Busse 1974), and $f \approx 3$ for $P r=0$ (Nagata \& Busse 1983)) have the same order of magnitude as in our case. Moreover, the origin of these oscillatory instabilities is also attributed in these works to the momentum advection terms, through the creation of vertical vorticity $\Omega_{z}$ (Clever \& Busse 1974; Nagata $\&$ Busse 1983). The rolls oscillate both horizontally and vertically (Meneguzzi et al. 1987). As in our case, a negative $y$-vorticity roll moves down when moving in the $x$-direction. This can in fact be related to the deformation term $\boldsymbol{\Omega} \cdot \boldsymbol{\nabla u}$ in the vorticity equation (Batchelor 1967): for a steady roll corresponding mainly to negative $\Omega_{y}$, a velocity field such that $(\partial u / \partial y)<0$ (corresponding to positive $\Omega_{z}$ ) will lead to the creation of positive $\Omega_{x}$.

\subsection{Comparisons between two- and three-dimensional results}

The two-dimensional results correspond to the $4 \times 1$ rigid cavity and the threedimensional results to the $4 \times 2 \times 1$ rigid cavity. In both situations we find a concentration of the main roll in the cell corresponding in a smooth way to the onset of the multi-roll structure: this transition occurs quite quickly in the two-dimensional case and leads to the subsequent creation of two end vortices; it is delayed in the three-dimensional case and no such end vortices appear.

The transition to oscillatory convection corresponds in these two- and threedimensional cases to values of $\mathrm{Gr}$ (and frequency) not very different (for more confined three-dimensional cases as the $4 \times 1 \times 1$ cavity, the threshold is strongly increased at least up to 135000 as obtained experimentally by Pratte \& Hart (1990)). These transitions both have a dynamical origin, but they occur in a different way in the two situations: in the two-dimensional case the oscillations correspond to successive expansions and recessions of the three rolls in the cavity; in the threedimensional case the oscillations have a three-dimensional character and correspond to the bending of the roll present in the cell in the form of a standing wave. The variations of the flow in the $V_{l}$ plane are very weak and cannot compare with the two-dimensional results. 
Finally, concerning the frequency, the comparisons are difficult because of the different nature of the oscillations: the main result is that the variation of the frequency with the Prandtl number is stronger in the three-dimensional case than in the two-dimensional case.

\section{Conclusion}

The convective flows, which arise in shallow cavities filled with low-Prandtl-number fluids when subjected to a horizontal temperature gradient, have been studied numerically with a finite element method. In order to allow a detailed analysis of the phenomena, attention has been focused on a rigid cavity with dimensions $4 \times 2 \times 1$ for which experimental data were available. The study has been performed in both two-dimensional and three-dimensional cases.

The two-dimensional results at $P r=0$ give a first steady transition to a multi-roll structure followed by a second transition to a mono-periodic flow characterized by pulsations of the rolls. The three-dimensional results at $P r=0$ show a similar but slower steady evolution corresponding to the concentration of the initial Hadley circulation into a large roll in the core of the cavity. The further transition to timedependent flows occurs as a three-dimensional roll oscillation corresponding to the creation of vertical and longitudinal vorticity. This transition is a Hopf bifurcation that breaks some symmetries of the steady flow, but preserves them between states separated by half the period. This behaviour is associated to the anti-symmetric character of the perturbations, and is shown to be a general result for such dynamical systems. This transition is preferred to the transition of two-dimensional type without breaking of symmetry. In these oscillatory flows the production of fluctuating kinetic energy comes principally from shear of the mean flow.

Calculations performed in the case of mercury $(P r=0.026)$ give similar results which compare well with the experimental data: more precisely, the experimental time series and power spectra of the temperature show typical properties related to the symmetries observed in the calculations. This validates the computational results and the analysis of the nature of the global flow and of the symmetry properties. Regarding the influence of a non-zero $P r$ value, the main effects are the increase of the oscillatory threshold and the strong increase of the frequency.

Finally, these results have been discussed with respect to previous works. The roll oscillations obtained in our study compare with those obtained numerically or by stability analyses in horizontal or inclined layers of low- $P r$ fluids heated from below where an array of parallel rolls has developed.

This study has focused on a rigid cavity with dimensions $4 \times 2 \times 1$. It would be interesting to investigate other aspect ratios in order to determine the domain of existence of the oscillatory instability which has been found, and perhaps other types of instabilities. However, such three-dimensional simulations remain computationaly expensive and may require the development of new algorithms.

\section{REFERENCES}

Afrid, M. \& ZeBIB, A. 1990 Oscillatory three-dimensional convection in rectangular cavities and enclosures. Phys. Fluids A 2, 1318-1327.

Batchelor, G. K. 1967 An Introduction to Fluid Dynamics. Cambridge University Press.

Ben HAdid, H. \& Roux, B. 1987 Oscillatory buoyancy-driven convection in horizontal liquid-metal layer. In Proc. VIth European Symposium on Materials and Fluid Sciences in Microgravity (ESA SP-256), pp. 477-485. ESA Publ. Division c/o ESTEC, Noordwijk, The Netherlands. 
Bontoux, P., Roux, A., Fontaine, J.P., Randriamampianina, A., Extremet, G. P., Crespo del Arco, E. \& Pulicani, J.P. 1990 Complex (and time-dependent) natural convection in lowPrandtl-number melts. In Progress in Astronautics and Aeronautics, vol. 130. AIAA.

Braunsfurth, M. G. \& Mullin, T. 1996 An experimental study of oscillatory convection in liquid gallium. J. Fluid Mech. 327, 199-219.

BufFAT, M. 1991a Simulation of two and three-dimensional internal subsonic flows using a finite element method. Intl J. Num. Meth. Fluids 12, 683-704.

Buffat, M. $1991 b$ Etude de la simulation numérique par une méthode d'éléments finis des écoulements internes subsoniques instationnaires bi et tridimensionnels. Thesis, 92-02, Université Claude Bernard, Lyon1.

Buffat, M. \& Henry, D. 1991 Numerical simulation of three-dimensional instabilities in liquid metal by a finite element method. In Numerical Methods in Thermal Problems, vol. VII, Part 1 (ed. R. W. Lewis, J. H. Chin \& G. M. Homsy), pp. 488-498. Pineridge Press, Swansea, UK.

Busse, F.H. \& Clever, R. M. 1981 An asymptotic model of two-dimensional convection in the limit of low Prandtl number. J. Fluid Mech. 102, 75-83.

Clever, R. M. \& Busse, F.H. 1974 Transition to time-dependent convection. J. Fluid Mech. 65, 625-645.

Clever, R. M. \& Busse, F. H. 1981 Low Prandtl number convection in a layer heated from below. J. Fluid Mech. 102, 61-74.

Cormack, D. E., Leal, L. G. \& Imberger, J. 1974 Natural convection in a shallow cavity with differentially heated endwalls. J. Fluid Mech. 65, 209-230.

Crochet, M. J., Geyling, F. T. \& Van Schaftingen, J. J. 1983 Numerical simulation of the horizontal Bridgman growth of a gallium arsenic crystal. J. Cryst. Growth 65, 166-172.

Crochet, M. J., Geyling, F. T. \& Van Schaftingen, J. J. 1987 Numerical simulation of the horizontal Bridgman growth. Part 1: two-dimensional flow. Intl J. Num. Meth. Fluids 7, 29-47.

Deane, A. E., Kevrekidis, I. G., Karniadakis, G. E. \& Orszag, S. A. 1991 Low-dimensional models for complex geometry flows: Application to grooved channels and circular cylinders. Phys. Fluids A 3, 2337-2354.

Drummond, J. E. \& Korpela, S. A. 1987 Natural convection in a shallow cavity. J. Fluid Mech. 182, $543-564$.

Dupont, S., Marchal, J. M., Crochet, M. J. \& Geyling, F. T. 1987 Numerical simulation of the horizontal Bridgman growth. Part 2: Three-dimensional flow. Intl J. Num. Meth. Fluids 7, 49-67.

GiLl, A.E. 1974 A theory of thermal oscillations in liquid metals. J. Fluid Mech. 64, 577-588.

HART, J. E. 1972 Stability of thin non-rotating Hadley circulations. J. Atmos. Sci. 29, 687-697.

HART, J. E. 1983a A note on the stability of low-Prandtl-number Hadley circulations. J. Fluid Mech. 132, 271-281.

HaRT, J.E. 1983b Low Prandtl number convection between differentially heated endwalls. Intl J. Heat Mass Transfer 26, 1069-1074.

Hart, J. E. \& Pratte, J. M. 1990 A laboratory study of oscillations in differentially heated layers of mercury. In Numerical Simulation of Oscillatory Convection in low-Pr Fluids (ed. B. Roux). Notes on Numerical Fluid Mechanics, vol. 27, pp. 338-343. Vieweg.

Henry, D. \& BufFAT, M. 1990 Computer simulation of the transition to oscillatory convection in highly conducting fluids heated from the side. A computer animation on a graphic workstation. First Liquid Matter Conference. 7-11 July 1990, Lyon (France).

Hung, M. C. \& ANDERECK, C. D. 1988 Transitions in convection driven by a horizontal temperature gradient. Phys. Lett. A 132, 253-258.

HuNG, M.C. \& ANDERECK, C.D. 1990 Subharmonic transitions in convection in a moderately shallow cavity. In Numerical Simulation of Oscillatory Convection in low-Pr Fluids (ed. B. Roux). Notes on Numerical Fluid Mechanics, vol. 27, pp. 338-343. Vieweg.

Hurle, D. T. J., Jakeman, E. \& Johnson, C. P. 1974 Convective temperature oscillations in molten gallium. J. Fluid Mech. 64, 565-576.

Iooss,G. 1988 Global characterization of the normal form for a vector field near a closed orbit. $J$. Diff Equat. 76, 47-76.

Iooss, G. \& JosePH, D. 1989 Elementary Stability and Bifurcation Theory, 2nd edn. UTM Springer.

KuO, H. P. \& Korpela, S. A. 1988 Stability and finite amplitude natural convection in a shallow cavity with insulated top and bottom and heated from a side. Phys. Fluids 31, 33-42. 
LAURE, P. 1987 Etude des mouvements de convection dans une cavité rectangulaire soumise à un gradient de température horizontal. J. Méc. Théor. Appl. 6, 351-382.

Laure, P. \& Roux, B. 1987 Synthèse des résultats obtenus par l'étude de stabilité des mouvements de convection dans une cavité horizontale de grande extension. C. R. Acad. Sci. Paris 305, $1137-1143$.

LE QUÉRÉ, P. 1990 Contribution to the GAMM workshop with a pseudo-spectral Chebyshev algorithm on a staggered grid. In Numerical Simulation of Oscillatory Convection in low-Pr Fluids (ed. B. Roux). Notes on Numerical Fluid Mechanics, vol. 27, pp. 227-236. Vieweg.

Mallinson, G. D. \& VAhl Davis, G. DE 1977 Three-dimensional natural convection in a box: a numerical study. J. Fluid Mech. 83, 1-31.

McKell, K. E., Broomhead, D. S., Jones, R. \& Hurle, D. T. J. 1990 Torus doubling in convecting molten Gallium. Europhys. Lett. 12, 513-518.

Meneguzzi, M., Sulem, C., Sulem, P. L. \& Thual, O. 1987 Three-dimensional numerical simulation of convection in low-Prandtl-number fluids. J. Fluid Mech. 182, 169-191.

Mundrane, M. \& Zebib, A. 1993 Two- and three-dimensional buoyant thermocapillary convection. Phys. Fluids A 5, 810-818.

Mundrane, M. \& Zebib, A. 1994 Oscillatory buoyant thermocapillary flow. Phys. Fluids A 6, 3294-3305.

NagATA, M. \& Busse, F. H. 1983 Three-dimensional tertiary motions in a plane shear layer. J. Fluid Mech. 135, 1-26.

OstRaCH, S. 1976 Convection phenomena at reduced gravity of importance for materials processing. In Proc. Second European Symposium on Materials and Fluid Sciences in Microgravity (ESA SP114), pp. 41-56. ESA Publ. Division c/o ESTEC, Noordwijk, The Netherlands.

PimputKar, S. M. \& Ostrach, S. 1981 Convective effects in crystals grown from melts. J. Cryst. Growth 55, 614-646.

Pratte, J. M. \& Hart, J.E. 1990 Endwall driven, low Prandtl number convection in a shallow rectangular cavity. J. Cryst. Growth 102, 54-68.

Pulicani, J.P., Crespo del Arco, E., Randriamampianina, A., Bontoux, P. \& Peyret, R. 1990 Spectral simulations of oscillatory convection at low Prandtl number. Intl J. Num. Meth. Fluids 10, 481-517.

Roux, B. (ED.) 1990 GAMM Workshop: Numerical Simulation of Oscillatory Convection in low-Pr Fluids. Notes on Numerical Fluid Mechanics, vol. 27. Vieweg.

Roux, B., Ben Hadid, H. \& Laure, P. 1989 Hydrodynamical regimes in metallic melts subject to a horizontal temperature gradient. Eur. J. Mech. B/Fluids 8, 375-396.

Roux, B., Bontoux, P. \& Henry, D. 1985 Numerical and theoretical study of different flow regimes occurring in horizontal fluid layers, differentially heated. In Macroscopic Modelling of Turbulent Flows (ed. U. Frisch, J. B. Keller, G. Papanicolaou \& O. Pironneau). Lecture Notes in Physics, vol. 230, pp. 202-217. Springer.

Sirovich, L. \& PARK, H. 1990 Turbulent thermal convection in a finite domain: Part I. Theory. Phys. Fluids A 2, 1649-1658.

Skeldon, A.C., Riley, D.C. \& Cliffe, K.A. 1996 Convection in a low Prandtl number fluid. J. Cryst. Growth 162, 95-106.

WANG, T. M. \& KoRpela, S. A. 1989 Convection rolls in a shallow cavity heated from a side. Phys. Fluids A 1, 947-953.

WANG, T. M. \& KORPELA, S. A. 1992 Secondary instabilities of convection in a shallow cavity. J. Fluid Mech. 234, 147-170.

WINTERS, K.H. 1988 Oscillatory convection in liquid metals in a horizontal temperature gradient. Intl J.Num. Meth. Engng 25, 401-414. 